1 Classification: Biological Sciences, Evolution

2

3 Title: Olfactory receptors tuned to volatile mustard oils in drosophilid flies

4

5 Authors: *Teruyuki Matsunaga ${ }^{1}$, ${ }^{*}$ Carolina E. Reisenman ${ }^{2},{ }^{*}$ Benjamin Goldman-Huertas ${ }^{3}$,

6 Philipp Brand ${ }^{4}$, Kevin Miao ${ }^{1}$, Hiromu Suzuki ${ }^{1}$, Santiago R. Ramírez ${ }^{4}$, Noah K. Whiteman ${ }^{1 \#}$

$7 \quad *$ Equal contributions

8

9 Affiliations:

101 Department of Integrative Biology, University of California Berkeley, Berkeley, CA

112 Department of Molecular and Cell Biology, University of California Berkeley, Berkeley, CA

123 Department of Molecular and Cellular Biology, University of Arizona, Tucson, AZ

134 Department of Evolution and Ecology, University of California Davis, Davis, CA

$14 \quad{ }^{\#}$ Correspondence to: whiteman@berkeley.edu (N.K.W.)

15

16 Keywords:

17 Scaptomyza flava, Drosophila melanogaster, herbivory, evolution, olfaction, isothiocyanate,

18 chemoreceptor, olfactory receptor, TrpA1, wasabi, Or67b

19 


\section{Abstract (248):}

22 Plant toxins are effective defenses because they are aversive to enemies. The same molecules,

23 however, are co-opted as host-finding cues by specialist herbivores. Although such behavioral

24 shifts are central to our understanding of herbivorous insect diversification, it is not well

25 understood how they evolve. We addressed this in Scaptomyza flava, a herbivorous drosophilid

26 fly within a lineage that shifted to feeding on toxic mustard plants (Brassicales) $<10$ million

27 years ago. S flava lost the ancestral attraction to yeast volatiles and the attendant

28 chemoreceptors that detect these odors. Here we report that $S$. flava, but not its close microbe-

29 feeding relatives Drosophila melanogaster and S. pallida, is attracted to mustard host-plant

30 odors, including volatile mustard oils (isothiocyanates or ITCs). Our genomic analysis uncovered

31 three $S$. flava paralogs of an olfactory receptor gene (Or67b) that likely experienced positive

32 selection. We then tested whether these chemoreceptors could underlie the observed attraction to

33 volatile ITCs. Our in vivo recordings revealed that two of the $S$. flava Or67b proteins (Or67b1

34 and Or67b3) - but not the homologous Ors from microbe-feeding relatives - responded

35 selectively and sensitively to volatile ITCs. These Ors are the only ITC chemoreceptors other

36 than TRP channel family members (e.g., the TrpA1 'wasabi' receptor) known from animals.

37 Remarkably, S. flava Or67b3 was sufficient to drive olfactory attraction toward butyl ITC when

38 expressed in an attractive olfactory circuit. Our study illuminates that ancestrally aversive

39 chemicals can be co-opted as attractants through gene duplication, leading to the origin of

40 hedonic valence shifts in herbivorous insects. 


\section{$44 \quad$ Significance Statement (120)}

45 Plant toxins trigger aversive olfactory (volatile-mediated) and gustatory (contact-mediated)

46 responses in animals. Paradoxically, toxic plants are colonized by specialist insects that co-opt

47 toxins as host-plant finding cues. The mechanisms underlying these behavioral shifts, from

48 indifference or repulsion, to attraction, remain unclear. To address this, we used a fly lineage,

49 Scaptomyza flava, that switched from yeast-feeding to feeding on mustard plants less than 10

50 million years ago. We found that S. flava is attracted to mustard-plant odors and volatile mustard

51 oils (isothiocyanates or ITCs) such as 'wasabi', a behavior enabled by the evolution of genes

52 encoding odorant receptors highly sensitive to ITCs. Our study illuminates how insects colonize

53 toxic host plants through duplication and ecological repurposing of genes encoding pre-existing

54 chemoreceptors.

55

56 
INTRODUCTION

Many plant compounds used in food, agriculture and medicine originally evolved as

60

61

62

63

64

65

66

67

68 toxins that deter and repel enemies (1). Among the most well-known compounds are those reactive electrophiles that induce the sensation of pain, such as diallyl disulfide and thiosulfinates produced by Alliaceae plants (e.g., onions and relatives), $\alpha, \beta$-unsaturated aldehydes found in Lauraceae (e.g., cinnamon and olives), and isothiocyanates (ITCs) from mustards and their relatives in the Brassicales. Paradoxically, herbivores specializing on Brassicales evolved electrophile diversion or resistance mechanisms and use these plants as a food source and habitat $(2,3)$. Mustard specialists can be trapped with ITC baits, indicating that these insects use "aversive" electrophiles for host finding (4). However, the mechanisms mediating the detection of species-specific electrophiles and underlying shifts in hedonic valence, are unknown.

In animals, the only known and broadly conserved electrophile detecting sensors are members of the Transient Receptor Potential (TRP) nociceptive channel family (5-7), and the most studied among them is the Transient Receptor Potential Ankyrin-1 (TrpA1) (8-10). Reactive electrophiles form covalent adducts with cysteine and lysine residues in TrpA1, activating this non-selective cation channel and resulting in the perception of pain (9). A reasonable hypothesis explaining the attraction of specialist mustard-plant insects to host-plantderived electrophiles is that the TrpA1 circuit has shifted from an aversive to an attractive one. However, TrpA1 is a gustatory, non-selective electrophile sensor activated by myriad electrophiles produced by plants in different orders, including diallyl disulfide, cinnamaldehyde, and ITCs, rendering this chemoreceptor ineffective in signaling host-plant identity (7).

Alternatively, chemoreceptor proteins (e.g., olfactory receptors -Ors- and/or ionotropic receptors -Irs-) that commonly mediate responses to volatile compounds in insects $(11,12)$ are better 
81 suited to evoke responses that signal volatile, host-specific electrophiles. Specificity for

82

83

84 ecologically relevant volatiles repeatedly arose through evolution of these olfactory chemoreceptors $(13,14)$. In $D$. sechellia, a specialist of the toxic "noni fruit", Ir75b evolved high sensitivity to a signature noni volatile through a single amino acid residue substitution (15). Similarly, in other drosophilid species, Or22a (and Or22b) evolved to be narrowly tuned to hostplant volatile esters $(16,17)$, facilitating host-plant specialization.

Here, we investigate the evolutionary and functional mechanisms underlying a major shift in host plant use: olfactory specialization on mustard plants by $S$. flava. S. flava is a relative of D. melanogaster nested phylogenetically in the paraphyletic Drosophila subgenus and is a specialist herbivore of ITCs-producing Brassicales plants $(18,19)$. In behavioral olfactory assays, we found that $S$. flava, but not its microbe-feeding relatives $S$. pallida or $D$. melanogaster, is attracted to volatile ITCs and odors from Brassicales plants. We scanned the genome sequence of $S$. flava to identify rapidly evolving chemoreceptors as candidates underlying this behavioral shift, and identified a lineage-specific expansion of three paralogous copies of the olfactory receptor gene $\operatorname{Or} 67 b$ that exhibited signatures of positive selection in the S. flava lineage. This $O r$, in contrast, is present as a single copy and is under strong evolutionary constraint in S. pallida and D. melanogaster. In vivo functional testing of Or67b copies from these species shows that two S. flava Or67b receptors, but not the conserved Or67b proteins from its microbe-feeding relatives, responded sensitively and selectively to volatile ITCs. Furthermore, D. melanogaster flies expressing S. flava Or67b3 were attracted to butyl ITC in olfactory assays, indicating that this receptor is sufficient for attraction to volatile ITCs in this context. We also found that ITCs are not detected through covalent modification of Or67bs, as opposed to TrpA1 (e.g. (7)). Or67b S. flava proteins selectively shifted their odor-receptive range 
104

105

106

107

108

109

110

111

112

113

114

115

116

117

118

119

120

121

122

123

124

125

to ITCs, an entirely new chemical class, from an ancestral olfactome of ketones, alcohols, and aldehydes. In summary, we discovered an evolutionary mechanism by which specialist herbivorous insects can shift hedonic valence from aversion to attraction towards host plantspecific electrophiles. A novel ITC-sensitive olfactory receptor system supplements the generalist TRP sensory system of a mustard specialist, an adaptation that evolved through gene duplication and neofunctionalization.

\section{RESULTS}

\section{S. flava is attracted to mustard plant odors and volatile ITCs}

Because $S$. flava is a Brassicales specialist, we first determined whether this species is attracted to mustard plant volatiles using a dual-choice olfactory assay (based on (20)). We found that both adult male and female $S$. flava are attracted to arugula (Eruca vesicaria) leaf odors (twotailed Binomial tests corrected for multiple testing using false discover rate (FDR), $<<0.05$; Fig. 1). In contrast, $D$. melanogaster was neither attracted nor deterred by arugula odors but instead was strongly attracted to apple cider vinegar volatiles $(F D R, p=0.0001)$, in agreement with previous results (e.g. (21)). In tests with apple cider vinegar, male $S$. flava preferred the odorless arm of the maze (FDR, $\mathrm{p}<0.05)$ and females showed a similar tendency (FDR, $\mathrm{p}=0.0541) . S$. pallida exhibited an intermediate behavior, distributing equally between the vinegar-laden and the odorless arms of the maze. Overall, these results demonstrate that $S$. flava, but not $D$. melanogaster or S. pallida, is attracted to mustard plant volatiles, and avoids odor sources that strongly attract $D$. melanogaster, characterized by the presence of acetic acid and a variety of ester, carbonyl, and hydroxyl-containing compounds $(22,23)$. 

relatives, is attracted to mustard plant volatiles and ITCs.

\section{Phylogenetic analysis identified $0 r 67 b$ paralogs as candidates mediating the detection of}

To identify potential $S$. flava chemosensory receptors involved in the attraction of and four other Drosophila species to identify Scaptomyza-specific losses and gains (Fig. 2A). 
PAML (26). Of 75 S. flava branches tested, seven branch models (for paralogs of Or63a, Or67b and $\operatorname{Or} 98 a$, and a homolog of $\operatorname{Or} 67 d$ ) indicated an $S$. flava foreground rate $>1$, consistent with

151 positive selection (Table S1). Of these receptors, homologs of Or67d detect the Drosophila pheromone 11-cis-vaccenyl acetate (27) and likely function in intraspecific communication in Scaptomyza, Or63a is only expressed in larvae (28), and the Or98a-like genes found in

154 Scaptomyza have no D. melanogaster homologs and are functionally uncharacterized. In contrast, Or67b is expressed in adults and modulates oviposition behavior (29), making it a good candidate. In a previous study, branch-site tests were also consistent with episodic positive selection acting on Or67b paralogs from $S$. flava (19). After expanding the representation of Or67b homologs in a phylogenetic analysis, and conducting branch tests in PAML on all branches in the tree, we found support for elevated $\mathrm{d} N / \mathrm{d} S$ exclusively in $S$. flava and $D$. mojavensis branches (Fig. 2B; TableS1). Thus, we focused on Or67b paralogs for functional 161 studies.

\section{S. flava Or67b proteins respond specifically to mustard plant volatiles}

We wondered whether any of the three $S$. flava Or67b proteins could mediate the odorspecific attraction of $S$. flava to mustard plant volatiles. First, we confirmed that all three across species, we used a D. melanogaster mutant that lacks its endogenous olfactory receptor (Or22a) in the ab3A olfactory receptor neuron (known as the "empty neuron system" $(30,31)$.

169 We conducted single sensillum recordings from the ab3 sensilla in transgenic D. melanogaster 170 flies expressing each of the S. flava (Sfla) Or67b paralogs, as well as the Or67b orthologs from

171 S. pallida (Spal) and D. melanogaster (Dmel) (Fig. 3A). Or22a deletion in ab3A was confirmed 
172 by the lack of responses in ab3A to ethyl hexanoate, a strong Or22a activator (Fig. S4A). We

173 found that ab3A neurons expressing any of the three Sfla Or67b paralogs responded to all

174 mustard plant volatiles tested (Fig. 3B; Mann-Whitney U tests adjusted for multiple comparisons

175 using FDR, $\mathrm{p}<0.05$ in all cases). In contrast, neurons expressing Dmel Or67b responded strongly

176 to apple cider vinegar (FDR, $\mathrm{p}<0.05)$, but not to any of the mustard plant volatiles tested (FDR,

$177 \mathrm{p}>0.1$ ). Neurons expressing Spal Or67b also showed strong responses when stimulated with

178 apple cider vinegar (FDR, $\mathrm{p}<0.05)$, but displayed only modest responses to arugula volatiles

179 (FDR, $\mathrm{p}<0.05)$. Interestingly, stimulation with apple cider vinegar decreased spiking activity of

180 neurons expressing Sfla Or67b1 (FDR, p<0.05).

Although neurons expressing any of the Sfla Or67b paralogs responded to mustard plant

volatiles, they differed in their responsiveness. Stimulation with arugula volatiles, for instance,

elicited stronger responses in neurons expressing Sfla Or67b1 (64 \pm 7.8 spikes/sec; average \pm

SE) than in neurons expressing Sfla Or67b2 (24 \pm 8.7 spikes/sec) or Sfla Or67b3 (22.3 \pm 2.3

$\mathrm{p}<0.05 ; \mathrm{n}=6$ in each group). In contrast, stimulation with horseradish volatiles produced stronger 


\section{S. flava Or67b paralogs respond selectively to ITCs and have different molecular receptive} ranges

Upon herbivore damage, mustard plants release ITCs and nitriles in addition to green leaf volatiles (GLVs) (32). To elucidate which odorants mediate activation of Sfla Or67b proteins, we tested a panel of 44 odorants which include ITCs, nitriles, GLVs, Dmel Or67b activators bearing various functional groups (33), and four ecologically relevant odorants which do not fall within these categories. Because many compounds were tested, for statistical comparisons we selected the odorants that produced the strongest average response across all Ors within each of the six chemical categories tested (those odorants were butyl ITC, mandelonitrile, cis-3-hexenyl butyrate, trans-2-pentenal, and geranyl acetate (Fig. $3 A$ and $C$ ). Neurons expressing any of the three $S$. flava paralogs responded to stimulation with butyl ITC (FDR, $\mathrm{p}<0.05)$, but not to stimulation with acetophenone (FDR, $\mathrm{p}>0.1)$. Conversely, acetophenone evoked the strongest excitatory responses from neurons expressing Dmel Or67b and Spal Or67b (respectively $175.7 \pm$ 17.6 and $78 \pm 15.2$ spikes/second; average \pm SE), while butyl ITC evoked no responses from neurons expressing Dmel Or67b (FDR, $\mathrm{p}>0.5$ ), and only produced a slight increase in spiking activity $(15 \pm 4.2$ spikes/second; average \pm SE; FDR, $\mathrm{p}<0.05)$ from neurons expressing Spal Or67b.

\section{S. flava Or67b1 and Or67b3 differ in their odor selectivity}

Although all S. flava paralogs are narrowly tuned to ITCs, they differ in their degree of selectivity and sensitivity. Butyl ITC evoked strong responses in neurons expressing Sfla Or67b1 and $\operatorname{Or67b3}(196.3 \pm 15.2$ and $170.6 \pm 11.2$ spikes/second; average \pm SE) that were not different from each other (Kruskal-Wallis ANOVA followed by Dunn's tests; $p>0.05$ ), while stimulation 
with this odorant produced only a modest response from neurons expressing Sfla Or67b2 (26.7 \pm 2.8 spikes/second; Dunn's tests, in both cases $\mathrm{p}<0.05)$. None of the 44 odorants tested evoked strong responses from neurons expressing Sfla Or67b2, but it is possible that other compounds not tested in this study, and in particular mustard plant volatiles (Fig. 3C), activate this receptor.

Next, because ITCs are highly diverse in structure (34), we hypothesized that a particular subset of ITCs differentially activate Sfla Or67b1 and Sfla Or67b3. In order to test this, we stimulated neurons expressing Sfla Or67b1 and Or67b3 with various concentrations of six ITCs (3-methyl-thio-propyl ITC, butyl ITC, isobutyl ITC, sec-butyl ITC, benzyl ITC, and phenethyl ITC) that evoked very strong responses (>123 spikes/second) from neurons expressing Or67b3 at 1:100 vol/vol (Fig. S4). At that concentration, only half of these odorants (3-methyl-thiopropyl ITC, butyl ITC, isobutyl ITC, and benzyl ITC) produced moderate to strong responses (41-147 spikes/second, depending on the odorant) from neurons expressing Sfla Or67b1. At 1:1,000 vol/vol, the responses of Sfla Or67b3 to stimulation with phenethyl ITC, sec-butyl ITC and butyl ITC were different from those of Sfla Or67b1 (Mann-Whitney U tests adjusted for multiple comparisons using FDR, $\mathrm{p}<0.05$ in all cases). Thus, although both Sfla Or67b1 and Or67b3 are highly selective and sensitive to ITCs, they have distinct odorant receptive ranges: Sfla Or67b3 is broadly tuned and strongly responds to all ITCs tested, while Sfla Or67b1 has a narrower receptive range and responds strongly only to a smaller subset of ITCs.

\section{Or67b response relatedness, specificities, and functional evolution}

We next compared the responses of all the Or67b proteins to all 44 odorants tested in a multi-dimensional space using principal component analysis (Fig. 4A). Each dot in Figure 4A shows the vectors quantifying the control-subtracted odor responses of each Or67b copy plotted 
along the dimensions of the first three principal components, which captured $63.1 \%, 26.0 \%$, and $9.0 \%$ of the variance of the original high-dimensional data set. This analysis shows that: 1) most

242 of the ITCs cluster separately from the other odorants tested, contributing to most of the variance

243 (PC1) of the data set; 2) some Dmel activators and GLVs such as acetophenone and cis-3-

244 hexenyl-butyrate also produced distinct responses, contributing largely to the variance of PC2; and 3) ITCs that activate Sfla Or67b1, such as sec-butyl ITC (but not butyl ITC) contribute to the variance of PC3. We also constructed tuning curves for each Or67b protein (Fig. 4B). The

247 strongest responses (center of the distribution) from Dmel Or67b and Spal Or67b were evoked by Dmel activators and GLVs, while the strongest responses from all Sfla Or67b paralogs were evoked by ITCs. Sfla Or67b1 is particularly narrowly tuned to a few ITCs, as revealed by the high kurtosis value of the distribution (Fig. 4B). Then we conducted a neighbor joining cluster analysis based on the Euclidean distances between the odor-evoked responses from all the Ors. Among the $S$. flava paralogs, Or67b1 and Or67b3 have the most similar (shorter Euclidean distance) odorant responses (Fig. 4C).

Activation of $S$. flava Or67b1 and Or67b3 requires the presence of the ITC functional

The finding that Sfla Or67b1 and Sfla Or67b3 respond to ITCs suggest that both the presence of the ITC functional group $(-\mathrm{N}=\mathrm{C}=\mathrm{S})$ and the size of the molecule determine binding

259 specificity. These two factors are indeed important to evoke responses from TrpA1, a non-

260 selective electrophile chemoreceptor that relies on ligand-directed covalent modification (10).

261 We addressed whether this is also the case for Sfla Or67b1 and Sfla Or67b3, by testing their

262 responses to eleven electrophiles (non-ITCs) that activate TrpA1 through covalent linkages with 
sulfhydryl side chains. Interestingly, none of the non-ITC electrophiles activated neurons expressing Sfla Or67b1, and among these, only trans-2-pentenal produced a very small increase in spike activity (4 \pm 1.3 spikes/second) from neurons expressing Sfla Or67b3 (Fig. 3C). Additionally, we found that neurons expressing Sfla Or67b can respond to odor pulses that are seconds apart (Fig. S5). Because recovery from ligand-induced covalent modifications takes at least several minutes (and often hours) (7), these results suggest that activation of Sfla Or67b1 and Or67b3 by ITCs does not rely on the TrpA1-like covalent modification strategy.

We next investigated whether the presence of the ITC functional group $(-\mathrm{N}=\mathrm{C}=\mathrm{S})$ is necessary for determining the responses of Sfla Or67b1 and Sfla Or67b3. In order to test this, we compared the responses of these Ors to benzyl ITC (BITC) and benzyl thiocyanate $(-\mathrm{S} \equiv \mathrm{C}-\mathrm{N}$ : BTC) (Fig.4B), linkage isomers of the same molecular weight. We found that BITC produced robust activity in neurons expressing Sfla Or67b1 or Sfla Or67b3 (Mann-Whitney U tests, in both cases $\mathrm{p}<0.002$ ), while BTC had little or no effect (Fig. $3 C$ and $4 B$ ). This differential activation pattern is likely due to the differences in the functional group because BITC and BTC are not only isomers, but also have similar volatilities. These findings are consistent with the hypothesis that Sfla Or67b1 and Sfla Or67b3 detect ITCs by interacting with the ITC $(-\mathrm{N}=\mathrm{C}=\mathrm{S})$ functional group selectively.

\section{S. flava Or67b3 is sufficient to induce olfactory attraction to ITCs}

Because Sfla Or67b paralogs, but not the orthologs from Spal and Dmel, specifically respond to ITC compounds, we asked whether these receptors can mediate olfactory attraction to these odor compounds. We focused on Sfla Or67b3, because this Or has a broader sensitivity to ITCs than Sfla Or67b1 (Fig. 4B). We investigated this by testing the behavioral responses of $D$. 
286

287

288

289

290

291

292

293

294

295

296

297

298

299

300

301

302

303

melanogaster "empty neuron" mutants that expressed Sfla Or67b3 or Dmel Or67b in the ab3A

neuron. As before, we used a dual-choice "Y-shaped" olfactometer offering an odorant stimulus

(butyl ITC 1:100 or 1:1,000 vol/vol) in one of the arms, and the solvent control in the other arm.

We found that at the 1:100 vol/vol concentration of butyl ITC the odor responses of flies

expressing Sfla Or67b3 or Dmel Or67b3 were different from the 50\% random expectation (twotailed Binomial tests, $\mathrm{p}<0.005$ and $\mathrm{p}<0.01$ respectively), with flies orienting towards the odor in ca. $70-71 \%$ of tests (Fig. 5). In contrast, at the lowest odor concentration (1:1,000 vol/vol), the odor responses of flies expressing Sfla Or67b3 - but not that of those expressing Dmel Or67bwere different from the 50\% random expectation (two-tailed Binomial tests, $\mathrm{p}<0.00005$ ), with flies choosing the odorous side of the maze in $83 \%$ of tests. Moreover, at the lower concentration, the odor-responses of flies expressing the $S$. flava transgene were different from those of flies expressing the Dmel transgene (Fisher Exact test, $\mathrm{p}<0.05$ ). None of the three genetic control Dmel lines showed a preference for either side of the maze (Binomial tests, $\mathrm{p}>0.1$ in all cases), even at the 1:100 vol/vol concentration. These results demonstrate that Sfla Or67b3 can confer odor sensitivity to ITCs that persists at low $(1: 1,000 \mathrm{vol} / \mathrm{vol})$ concentrations in a fly that is otherwise not attracted to these odor compounds.

\section{Discussion}

Plants have evolved a myriad of toxic compounds to deter enemies which are generally perceived as noxious by animals (1). In spite of this, the vast majority of herbivorous insect species are specialized on one or a few host-plant lineages that contain aversive compounds such as ITCs - the chemicals that give wasabi its characteristic pungent taste $(35,36)$. Through the course of evolution, specialist insects co-opted these chemicals as cues for host-finding, feeding, 
and egg laying (37). Here, we investigated how attraction to these ancestrally aversive chemical cues evolved, using S. flava, a recently-derived herbivorous fly of mustard plants and their relatives (Brassicales). In behavioral experiments we found that $S$. flava, but neither of its microbe-feeding close relatives $S$. pallida or D. melanogaster, is attracted to mustard-plant volatiles, including volatile ITCs (Fig. 1). We next searched for changes in chemoreceptor proteins that could underlie this host plant-specific olfactory attraction. We found a candidate olfactory receptor gene $($ Or67b), which was triplicated in a recent ancestor of $S$. flava, resulting in three divergent and paralogous odorant receptor genes (Sfla Or67b1-b3; Fig. 2). These Sfla Or67b paralogs evolved with rapid rates of amino-acid changing mutations, consistent with episodic positive selection (Fig. 2B; TableS1). Next, using the "empty neuron" system of $D$. melanogaster, we investigated the odor-response profiles of these receptors. Consistent with our behavioral results, we found that two of the Sfla Or67b paralogs (Sfla Or67b1 and Sfla Or67b3) specifically respond to stimulation with mustard-plant odors and volatile ITCs (Fig. 3 and Fig S5). In contrast, S. pallida and D. melanogaster paralogs responded strongly to stimulation with apple cider vinegar and a broad range of aldehydes, alcohols and ketones (Fig. 3 and Fig. 4), consistent with their microbe-feeding habits. We also found that the presence of the ITC functional group is necessary for activation of Sfla Or67b and furthermore, that activation of the receptor likely does not involve a TrpA1-like covalent modification (Fig. 3, Fig. 4 and Fig. S5 (7)). Remarkably, when Sfla Or67b3 was expressed in ab3A “empty neuron” system of $D$. melanogaster, adult female flies were attracted to butyl ITC (Fig. 5). This suggests that Sfla Or67b3 may be sufficient for the behavioral valence shift observed in the evolution of mustardfeeding Scaptomyza, when expressed in novel neural circuit that ancestrally mediates attraction. Our results reveal the first Ors characterized from any animal that are tuned to mustard oils, and 
332 illustrate an evolutionary mechanism by which mustard specialists can evolve attraction towards

333 otherwise aversive chemical compounds.

The gene triplication event that occurred in the $S$. flava lineage raises several interesting

molecular evolutionary questions. Gene duplication plays an important role in evolutionary innovation (38) and several outcomes have been proposed regarding to how gene duplication contributes to acquisition of novel gene functions $(38,39)$ : (A) neofunctionalization occurs when one of the two duplicated genes (paralogs) acquires a new function after accumulating de novo mutations, while the other copy retains its ancestral function; (B) subfunctionalization is largely considered a degenerative process whereby mutations accumulate in both copies, leading to

341 partitioning of the ancestral function between the two gene copies; (C) specialization occurs when subfunctionalization and neofunctionalization evolve simultaneously, yielding gene copies that are "functionally distinct from each other and from the ancestral gene" $(40,41)$. The

344 specialization model is supported by our data, as neither of the two outgroup Or67b proteins 345 (Spal Or67b and Dmel Or67b) responded to ITCs (neofunctionalization), coupled with the fact 346 that Sfla Or67b1 has a narrower breadth of tuning to ITCs than Sfla Or67b3

347 (subfunctionalization). Our conceptual model for this evolutionary hypothesis (Fig. 5) is that the ancestral Or67b (“1”) was broadly tuned to acetophenone, alcohols and ketones, and diverged

349 into a mustard oil-specific Or67b ("2") that lost sensitivity to these compounds and gained responsiveness to ITCs in the $S$. flava lineage. This Or triplicated during the course of evolution,

351 ultimately giving rise to three Ors with different odor selectivity (“3”): (a) Sfla Or67b3, which

352 retained broad sensitivity to ITCs; (b) Sfla Or67b1, which become narrowly tuned to a subset of 353 Sfla Or67b3 activators; and (c) Sfla Or67b2, which lost sensitivity to a broad range of ITCs (at 
354 least all the nine ITC compounds tested here) and likely retained or gained sensitivity to untested compounds.

Across Drosophila, orthologous chemoreceptors respond in a species-specific manner to ecologically relevant ligands. The best-studied of these is the Or22a gene, which has been evolutionarily deleted in $S$. flava but not in $S$. pallida (19). In several drosophilids, Or22a is tuned to different ecologically relevant esters: ethyl-hexanoate in D. melanogaster (16), methylhexanoate in D. sechellia (42), 3-methyl-2-butenyl acetate in D. erecta (17), and isobutyl acetate in D. suzukii (43). Similarly, Ir75b is tuned to butyric acid in D. melanogaster and to hexanoic acid in D. sechellia (15). The fact that the chemical classes are still conserved for each

363 chemoreceptor (i.e. esters in the case of Or22a and acids in the case of Ir75b), suggests that the

364 chemical niche space and underlying sensory mechanisms are relatively conserved across

365 species. In contrast, while Spal Or67b and Dmel Or67b respond to alcohols, aldehydes and ketones, Sfla Or67b1 and Sfla Or67b3 respond selectively to volatile ITCs, thus acquiring a previously undescribed sensitivity to an entirely different chemical compound class (Fig. 6b).

368 We hypothesize that the striking difference between Or67b-activating ligands among these species resulted from the dramatic shift from feeding on microbes to feeding on live mustard plant tissue that occurred in the evolution of herbivorous Scaptomyza.

372 chemoreceptors $(7,8,10)$. However, volatile ITCs are widely used to trap agricultural pests of

373 Brassicales (4), and in agreement with this, the antennae of some of these insects respond to

374 volatile ITCs (44). Our study further advances the understanding of how volatile ITCs may be

375 detected by these insects. Furthermore, we uncovered a potential evolutionary mechanism, gene 
377 Ors evolved in Brassicales specialists. More generally, our results suggest that major host shifts

378 and specialization events in herbivorous insects can result from simple genetic changes of large

379 effect in the peripheral nervous system, similar to the apple maggot fly Rhagoletis pomonella

380 (45). This in turn may explain why such events are so common across herbivorous insect

381 lineages, resulting in rapid rates of diversification and the most diverse guild of macroscopic life 382 to have evolved $(46,47)$.

Material and Methods:

Material and methods are summarized below. For complete details, see Supplemental Methods. Fly lines

M2-MD refers to a CRISPR/Cas9-mediated deletion of $O r 22 a / b$ and a knock-in of Gal4 and DsRed by homologous repair. Gal4 is not functional but DsRed is expressed in the eye. The functional absence of $\operatorname{Or} 22 a$ and $\operatorname{Or} 22 b$ genes in M2-MD flies was confirmed by electrophysiological analysis on Or22a/b expressing neurons in wild-type flies (Fig. S3). The four $U A S-O r 67 b$ lines created in this study, the UAS-Dmel Or67b line, and the UAS-dTrpA1 (stock no. 26264) line was each crossed into the M2-MD line. The progeny were then used for SSR recordings and behavioral experiments.

\section{Behavioral tests of olfactory attraction}

The olfactory responses of mated, non-starved D. melanogaster (Canton-S or transgenic), S. pallida, and S. flava were tested using a dual-choice "Y-shaped" olfactometer based on one previously published (20). Insects could walk upwind towards the "decision point" (intersection 
400 of the short and long arms of the "Y") and turn towards either the odor-laden or the odorless arm

401 of the maze. Four insects were released at once (to increase experimental efficacy), but only the

402 first choice was considered.

403

The odorants (20 $\mu 1$ of 1:100 vol/vol mineral oil solution) used in experiments were butyl

ITC (Sigma-Aldrich, CAS \# 592-82-5, USA) and sec-butyl ITC (Sigma-Aldrich, CAS \# 15585-

405

98-5, USA). For tests with transgenic flies, responses were tested at 1:100 vol/vol and 1:1,000

406

vol/vol of butyl ITC. We also used apple cider vinegar (40 $\mu$ l, O Organics, USA; $40 \mu 1$ of

407 distilled water was as control stimulus in these tests). For tests of host orientation, two-four

greenhouse were excised just before tests.

410

For each odor/odorant, species, sex and genotype, the number of tests with insects

orienting towards the odorous arm and the odorless arm of the Y-maze was used to conduct two-

412 tailed Binomial tests (48). When appropriate, p-values were adjusted for multiple

413 testing/comparisons using the false discovery rate (FDR) method of Benjamin-Hochberg (49);

414 results were considered statistically significant if the Benjamini-Hochberg adjusted p-value was

$415<0.05$. For all tests, we verified that the power was $>0.8$.

417 Molecular phylogeny of drosophilid odorant receptors:

Translations of Ors from D. grimshawi, D. mojavensis, D. virilis and D. melanogaster

419 (builds dgri r1.3, dmoj r1.3, dvir r1.07 and dmel r6.28, respectively) were downloaded from

420 Flybase (www.flybase.org, (50)) and S. flava sequences were obtained from ref. (19). Three

421 hundred and nine sequences were aligned in MAFFT v7.017 (51). Models were fitted to the

422 alignment using IQ-Tree and tested using the AIC criterion (52). A maximum likelihood (ML) 
423 phylogeny was generated using the Or protein alignment in RAxML v8.2.10 (53). Orco

424 sequences were designated as the outgroup.

425

426 Molecular phylogeny of drosophilid Or67b genes:

427 Or67b CDS from D. grimshawi, D. mojavensis, D. virilis D. sechellia, D. simulans, D. erecta, D.

428 yakuba, D. pseudoobscura, D. persimilis, D. ananassae, D. willistoni and D. melanogaster

429 (builds dgri r1.3, dmoj r1.3, dvir r1.07, dsec r1.3, dsim r1.4, dere r1.3, dyak r1.3, dpse r3.2, dper

430 r1.3, dana r1.3, dwil r1.3 and dmel r6.28, respectively) were downloaded from Flybase

431 (www.flybase.org, (50)). The $S$. pallida DNA sequence was obtained through PCR. Sequences

432 were aligned, models fitted and chosen according to AIC (GTR+I+G) in IQ-Tree (52). Trees

433 were inferred using RAxML (v8.2.10) with the GTRCATI model and 1000 rapid bootstraps, and

434 MrBayes (v3.2.6) (54).

436 Analysis of molecular evolution:

437 CDS of homologs of every Or gene in S. flava found in the 12 Drosophila genome builds were

438 aligned to S. flava Or CDS. Homology was assessed according to inclusion in well supported

439 clades in the Or translation phylogeny above. Sequences were aligned in MAFFT (v7.017) (51).

440 Phylogenies were generated for every alignment using PhyML (55). If these trees showed $>70 \%$

441 bootstrap support for a topology contrary to the known species topology, or if the Or homology

442 group contained duplicates, these trees were used in PAML analyses instead of the species tree.

443 Branch models of sequence evolution were fit using PAML 4.9h (26). A foreground/background

444 branch model was fit for every S. flava tip branch and every ancestral branch in a Scaptomyza- 
445 specific Or gene duplication clade, and compared in a likelihood ratio test to a null model with

446 one $\mathrm{d} N / \mathrm{d} S$ rate for every unique phylogeny (total: 75 tests). After focusing on Or67b, patterns of

447 molecular evolution among the drosophilid Or67b homologs were explored using the expanded

448 Or67b CDS phylogeny above. Foreground/background branch models were fit for every branch

449 in the Or67b phylogeny with identical likely ratio tests performed as above (29 tests total Fig.

$4502 B$; Table S1). P-values were adjusted for multiple testing using the FDR method (49).

\section{Distance tree based on SSR recordings from neurons expressing Or67b transgenes}

453 A matrix of average responses from five $0 r 67 b$ transgene receptors to 44 odorant compounds

454 (1:100 vol/vol concentration) was produced (Table S3). Net responses were obtained by

455 subtracting the response to mineral oil or dimethyl sulfoxide solvents. A Euclidean distance

456 matrix was generated using the dist function from the $\mathrm{R}$ stats package (56). Receptor responses

457 were clustered by using the neighbor joining (NJ) algorithm on this distance matrix. Support for

458 clusters was assessed using 1,000 bootstraps of the original response matrix by generating

459 distance matrices and NJ trees on the pseudo-datasets in ape (v5.3) (57).

\section{Single sensillum recordings (SSR)}

462 Adult female flies were fed and then prepared for SSR as previously described (58).

463 Extracellular activity was recorded by inserting a tungsten electrode into the base of the ab3

464 sensillum. The following odor sources (all purchased in Berkeley, California, US unless

465 otherwise mentioned; $20 \mu \mathrm{l}$ of material loaded on filter paper) were used: apple cider vinegar $(\mathrm{O}$

466 Organics, USA), grated roots of Wasabia japonica (wasabi), organic roots of Armoracia

467 rusticana (horseradish), Brassica rapa (turnip), Raphanus sativus (daikon), and Beta vulgaris 
468 (beet). Eruca vesicaria (arugula) was grown from seeds in the lab, and leaves of 3-8 weeks old

469 plants were used for odor stimulation. Roots or leaves were grated and homogenized using a

470 vegetable grater to a volume equivalent to $\sim 500 \mu 1$. The "net number of spikes" were obtained by

471 counting the number of spikes during a 1-second window 0.2 seconds after the onset of

472 stimulation, and subtracting from this number the background spiking activity (number of spikes

473 during 1-second previous to the onset of odor stimulation). Data were analyzed using Mann-

474 Whitney Rank sum tests (for comparisons involving two means) and p-values were adjusted for

475 multiple comparisons using the FDR method, or by Kruskal-Wallis ANOVAs followed by

476 Dunn's or Dunnet's tests (for comparisons involving more than two means).

\section{Data Analysis and figure generation:}

479 All images and drawings are originals prepared by the authors. Figures were prepared via a combination of WinEDR (v3.9.1), R Studio (v1.2.1335), Microsoft Excel (2016), Adobe

481 Illustrator (2019), Python, and Geneious (10.0.9).

\section{Acknowledgments:}

484 We are grateful to Drs. Chauda Sebastian, Dennis Mathew, John Carlson and Bloomington

485 Drosophila Stock Center (NIH P40OD018537) for sharing M2-MD, UAS-Dmel Or67b, and UAS-

$486 d \operatorname{Trp} A 1$ (stock no. 26264), and to Drs. Johannes Bischof and Konrad Basler for donation of the

487 pUASTattB plasmid. C.E.R. thanks Dr. Kristin Scott for support and encouragement. We thank 
490 the National Institutes of Health (award number R35GM119816 to N.K.W.) and the National

491 Science Foundation (award number IOS 1755188 supporting B.G.-H.).

492

493

494

495

496

497

498

499

500

501

502

503

504

505

506

507

508

509

510

511

512

513

514

515

516

517 
bioRxiv preprint doi: https://doi.org/10.1101/2019.12.27.889774; this version posted December 30, 2019. The coply gihtholderforthispreprint (which was not certified by peer review) is the author/funder, who has granted bioRxiv a license to display the preprint 0 pelpetuty. It made

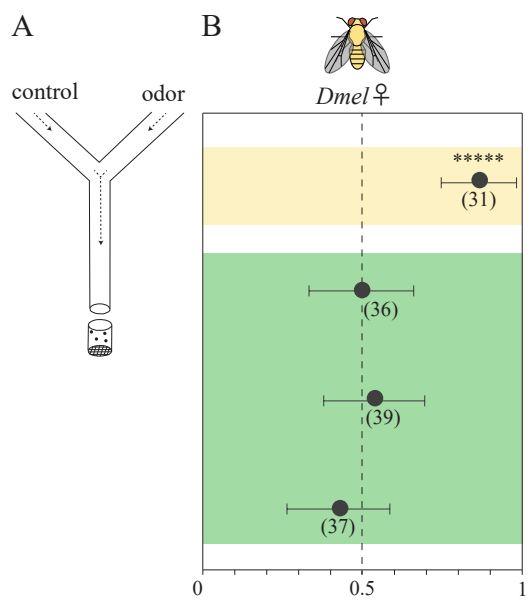
available under aCC-BY-ND 4.0 International license.
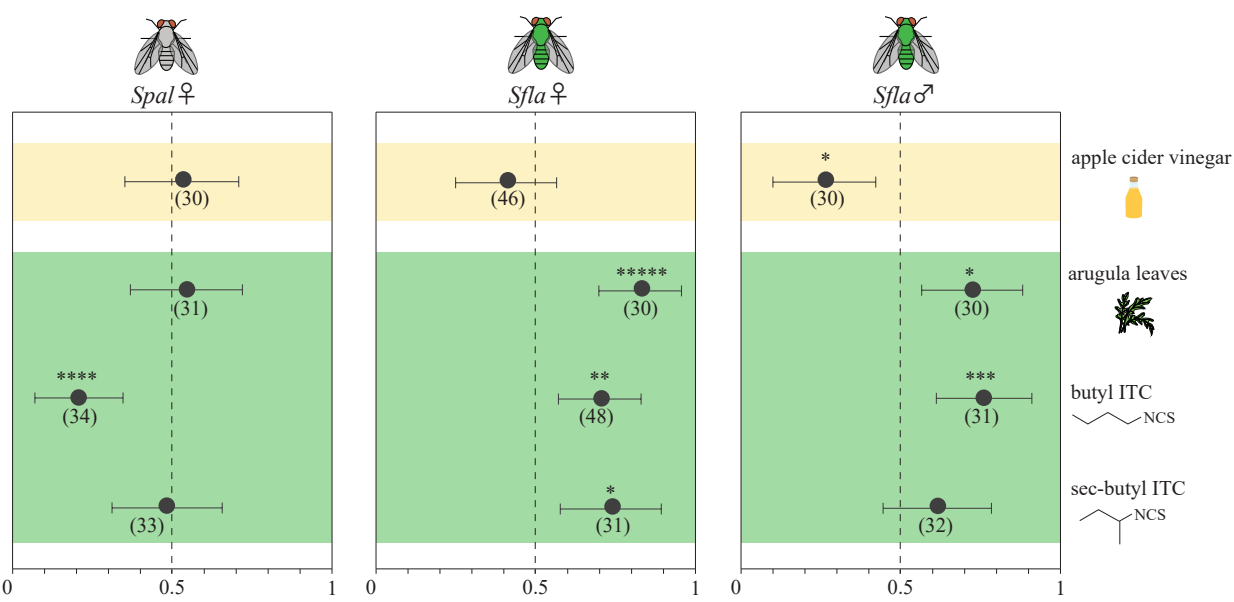

Proportion of tests with orentation towards the odor 


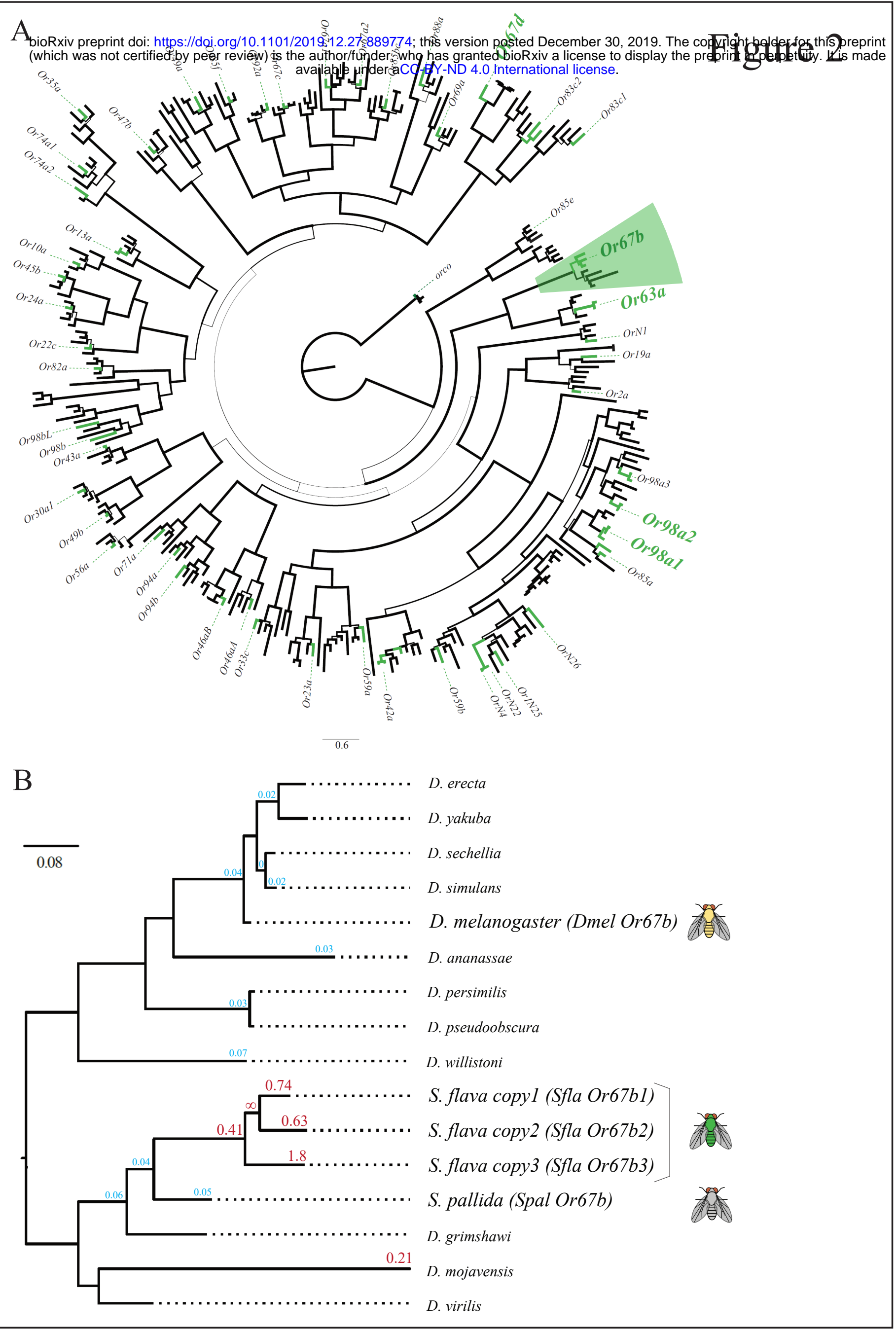




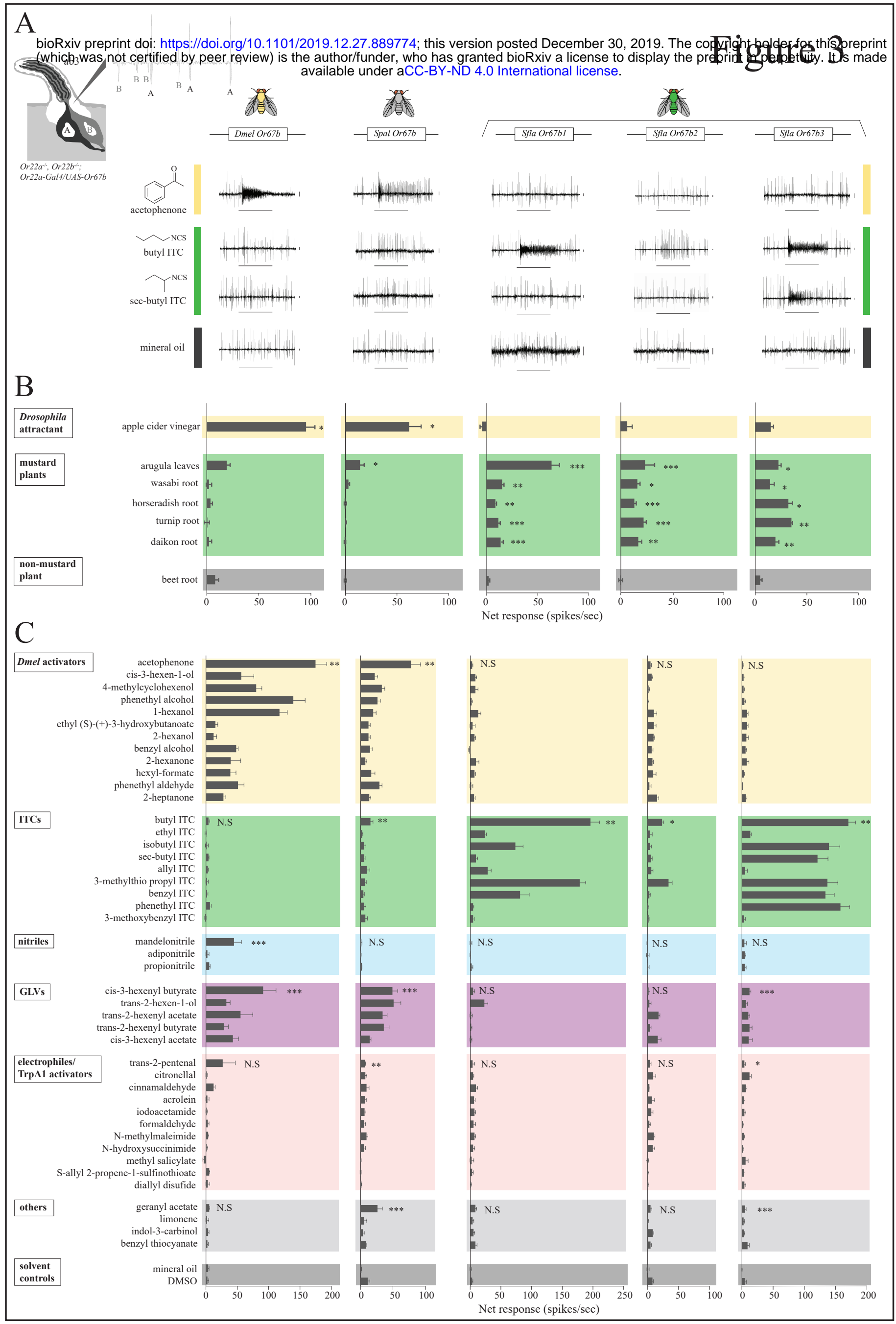


bioRxiv preprint doi: https://doi.org/10.1101/2019.12.27.889774; this version posted December 30, 2019. The coplylghtholderforthis/preprint $\mathrm{A}$ (which was not certified by peer review) is the author/funder, who has granted bioRxiv a license to display the preprint ${ }^{2}$ perpetuty. ttis made available under aCC-BY-ND 4.0 International license.
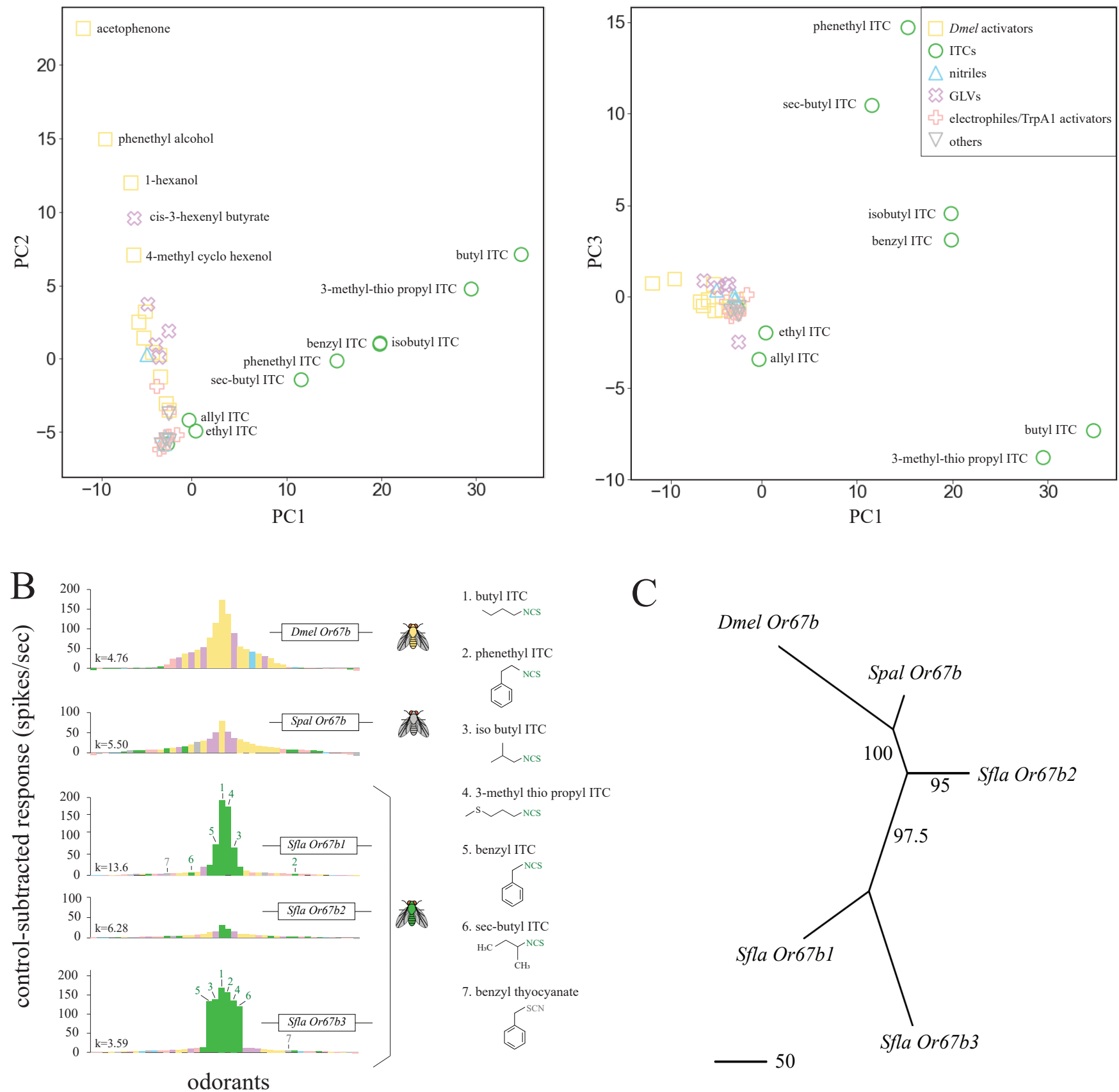


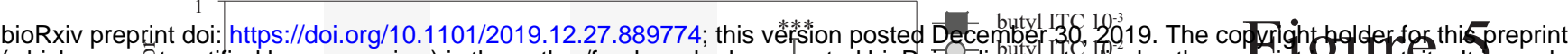
(which was thertified by peer review) is the author/funder, who has granted bioRxiva license to display the preprint @pelpetuty. It made available under a:CC-BY-ND 4:a* *aternational license.

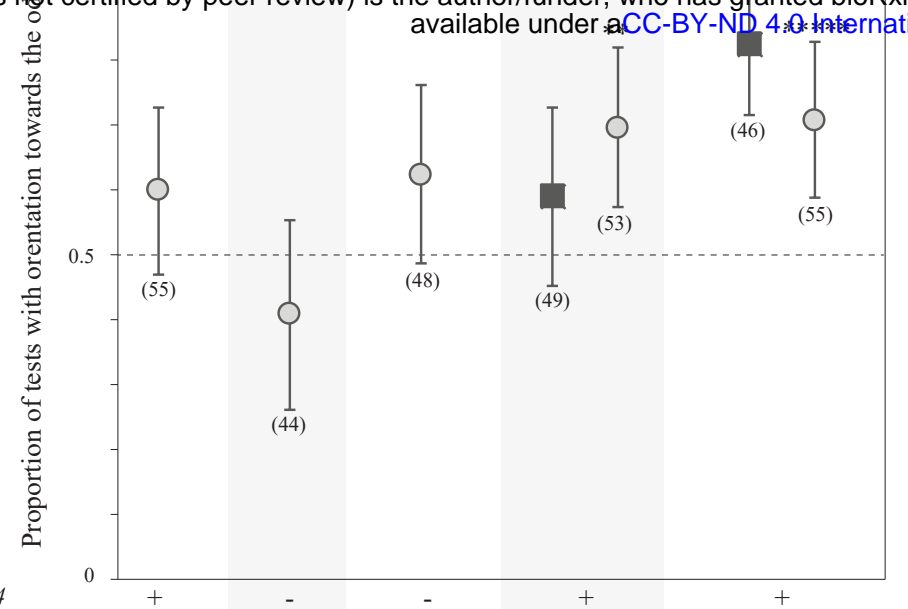

Or22a-Gal4

UAS-Dmel Or67b

UAS-Sfla Or67b3 

AbioRxiv preprint doi: https://doi.ßrg/10.1101/2019.12.27.889774; this version posted December 30, 2019. The copyrightholderforthifpreprint
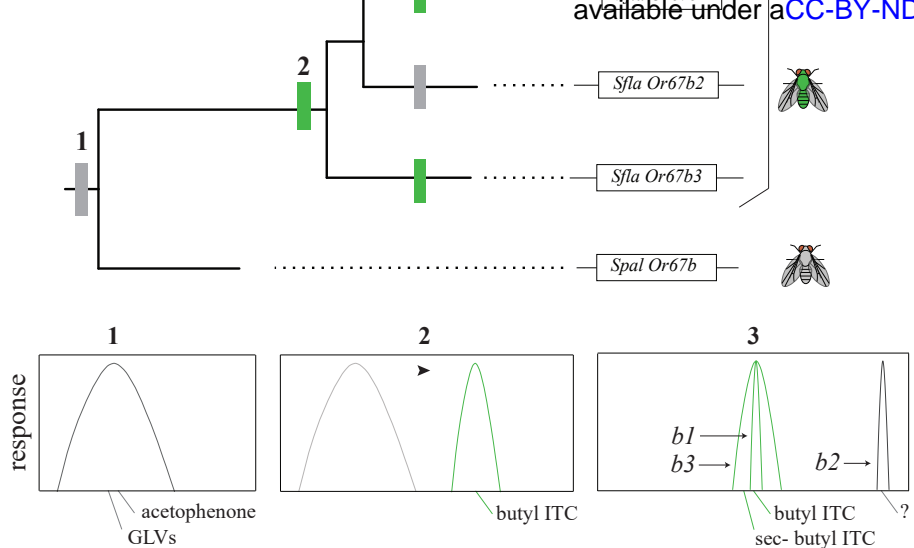

odorants

B

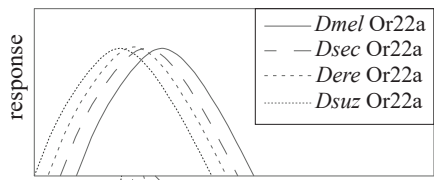

ethyl hexanoate

methyl hexanoate

3-methyl-2-butenyl acetate

iso butyl acetate
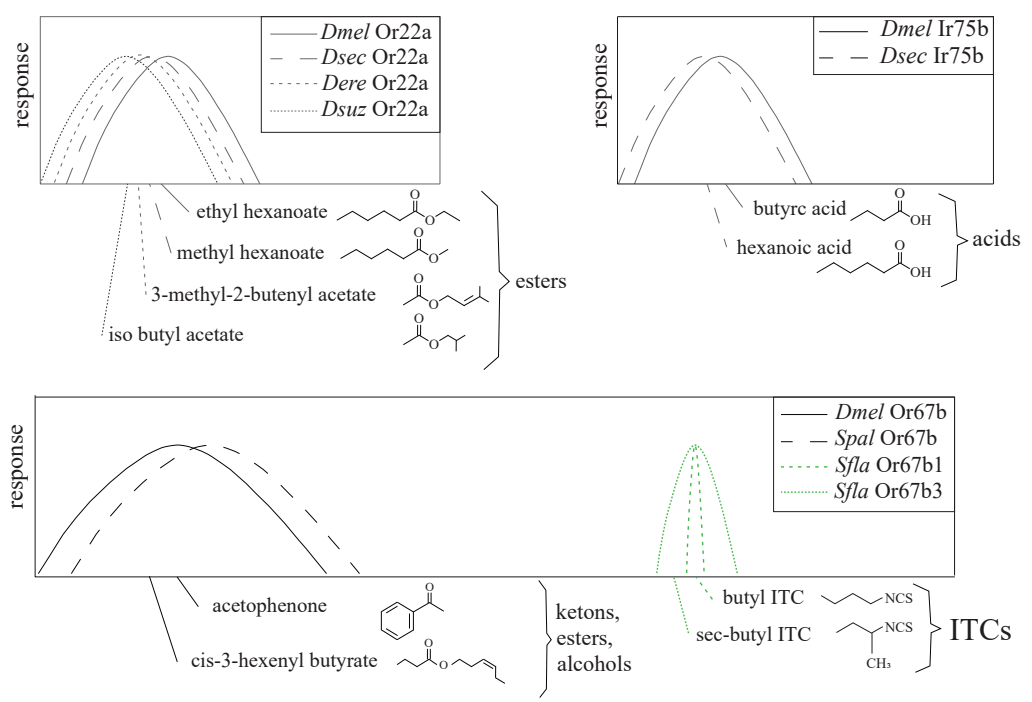


\section{Figure Legends:}

Figure 1: Olfactory behavioral responses of $S$. flava and its microbe-feeding relatives $S$. just before tests and placed in a 5-ml syringe (the control syringe contained clean tissue paper).

other arm of the maze. (B) Olfactory behavioral responses of D. melanogaster, $S$. pallida and $S$.

532 flava to the odors and odorants indicated to the right. Data represent the proportion of tests in

which insects choose the odorous arm of the maze, and the error bars indicate the $95 \%$

confidence interval of the proportion; the dotted reference line at 0.5 indicate no preference for 
542 Figure 2: Maximum likelihood (ML) phylogeny of Or67bs in Drosophilidae. (A)

543 ML phylogeny reconstructed from protein translations of the Ors found in S. flava, D.

544 melanogaster, D. grimshawi, D. virilis and D. mojavensis genomes. Line width of branches are

545 proportional to bootstrap support. Green branches indicate Scaptomyza Ors. Gene names with

546 bold fonts include branches with estimated $\mathrm{d} N / \mathrm{d} S>1$ from branch tests conducted in PAML,

547 consistent with episodic positive selection. (B) ML phylogeny reconstructed from coding

548 sequence of Or67b orthologs of twelve Drosophila species, S. pallida and S. flava. All bootstrap

549 supports for the nodes are $>80 \%$ and all posterior probabilities were $>0.95$ for the MrBayes tree.

550 Branches with significant support (FDR p-value $<0.05)$ for $\mathrm{d} N / \mathrm{d} S$ values different from the

551 background rate are indicated with colored branch labels (blue where the foreground rate is less

552 than the background, and red where $\mathrm{d} N / \mathrm{d} S$ is greater than the background). Only $S$. flava and $D$.

553 mojavensis branches have significantly elevated $\mathrm{d} N / \mathrm{d} S$ according to branch model tests. $S$.

554 flava, S. pallida and D. melanogaster branches are indicated by fly icons colored as in Figure

555 1. Scale bars are in units of substitutions per site.

556

557

558

559

560

561

562

563 
Figure 3: Odor responses of orthologous and paralogous Or67b from D. melanogaster, $S$.

566

567

568

569

570

571

572

573

574

575

576

577

578

579

580

581

582

583

584

585

586

587 pallida and S. flava to mustard-plant volatiles.

(A) Or67b paralogs and orthologs were expressed in a D. melanogaster mutant that lacks its endogenous $\operatorname{Or}(\operatorname{Or} 22 a)$ in the ab3A olfactory receptor neuron (30). Shown is a representative trace of a single sensillum recording obtained from a D. melanogaster fly with Dmel Or67b expressed in the ab3A neuron (Or22a $a^{-/}$Or22b $^{-/-}$; Or22a-Gal4/UAS-Dmel Or67b) (top left). Recordings from the ab3 sensilla produced spike trains from the two olfactory receptor cells housed within this sensilla (ab3A, which expresses $\operatorname{Or} 67 \mathrm{~b}$, and ab3B, which expresses the native Or85b) that had distinct spike amplitudes. The ab3A neuron (labeled A) produced spikes of larger amplitude than the ab3B neuron (labeled B). Scale bar: $10 \mathrm{mV}$. Bottom: representative electrophysiological recordings obtained from the ab3 sensilla of flies expressing Dmel Or67b, Spal Or67b, Sfla Or67b1, Sfla Or67b2 or Or67b3 in the ab3A neuron in response to stimulation with acetophenone, butyl ITC, sec-butyl ITC and mineral oil (solvent control). The bars below records indicate the onset and duration $(1 \mathrm{sec})$ of the odor stimulation. Calibration bars: $10 \mathrm{mV}$. (B) Responses (net number of spikes/second; average \pm SE, $n=6-8$ sensilla obtained from 2-4 females) evoked by stimulation with apple cider vinegar, mustard plant volatiles and nonmustard plant volatiles (beet, control). Asterisks indicate significant differences between the responses to stimulation with an odor and the control stimulus (beet) (Mann-Whitney U tests corrected for multiple comparisons using FDR; adjusted $\mathrm{p}$-values: $* \mathrm{p}<0.05 ; * * \mathrm{p}<0.01 ; * * *$ $\mathrm{p}<0.005)$. Note that neurons expressing Dmel Or67b and Spal Or67b, but not those expressing any of the $S$. flava Or67b paralogs, respond to apple cider vinegar. Conversely, only neurons expressing $S$. flava Or67b paralogs respond to mustard plant volatiles; neurons expressing Sfla Or67b3 responded to all mustard plant volatiles tested. (C) Responses (net number of spikes/sec; 
588

589

590

591

592

593

594

595

596

597

598

599

600

601 GLVs.

602

603

604

605

606

607

608

609

(1)

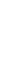

(1)

.

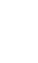

(1)

(1)

average $\pm S E, n=6-20$ for each odorant, $n=6-15$ for mineral oil, obtained from 2-7 females)

evoked by stimulation with single odorants categorized as follows: Dmel Or67b activators

(Database of Odor Responses, (33); orange), ITCs (green), nitriles (blue), green leaf volatiles

(GLVs, purple), electrophiles/TrpA1 activators (pink), other odorants not belonging to any of the previous categories (gray), and the two solvent controls (black). The responses of the strongest activator within each category (acetophenone, butyl ITC, mandelonitrile, cis-3-hexenyl butyrate, trans-2-pentenal, and geranyl acetate) were compared against the mineral oil control. Asterisks indicate significant differences between the responses to stimulation with each of these odorants and the control stimulus (statistics as described in B). Note that ITCs strongly activate neurons expressing Sfla Or67b1 or Sfla Or67b3, and that for the most part these Ors do not respond to any of the other odorants tested. Some ITCs, such as sec-butyl ITC and phenethyl ITC, only activated neurons expressing Sfla Or67b3. Spal Or67b has an odorant response profile similar to that of Dmel Or67b, responding mostly to stimulation with D. melanogaster activators and 
Figure 4: Or67b orthologs and paralogs have distinct and non-overlapping odor-response

611 profiles, tuning breadths, and functional relatedness. (A) Vectors quantifying the odor-

612 evoked responses (control-subtracted) were projected onto a two-dimensional subspace

613 determined by the first three principal components of the data-set (left: PC1 vs PC2; right: PC1

614 vs PC3). PC1-3 captured each $63.1 \%, 26.0 \%$, and $9.0 \%$ of the variances of the original five-

615 dimensional data. Each dot represents one odorant, and the different colors and symbols indicate

616 different chemical categories (as in Figure 3C and in the inset, right graph). (B) Tuning curves of

617 each Or67b copy, showing the distribution of mean responses $(n=6-20)$ to the 44 odorants tested

618 (color-coded as in A). The odorants are displayed along the horizontal axis according to the

619 responses (net spikes/sec) they elicit from each Or. The odorants eliciting the strongest responses

620 for each Or are located at the center of the distribution, while weaker activators are distributed

621 along the edges of the distribution. Thus, the order of the odorants is different for the five Ors.

622 Note that the strongest responses (center of the distribution) from Dmel Or67b and Spal Or67b

623 are evoked by D. melanogaster activators and GLVs, while the strongest responses from all Sfla

624 Or67b paralogs are evoked by ITCs. The tuning breadth of each Or is quantified by the kurtosis

625 value $(\mathrm{k})$ of the distribution with higher values indicating narrower odor-response profiles. The

626 chemical structure of the top six Sfla Or67b3 activators and benzyl thiocyanate (numbers in

627 green) are indicated to the right. (C) Neighbor joining cluster analysis for the five Or67b

628 orthologs based on the Euclidean distances between the odor-evoked responses of the receptors.

629 Dmel Or67b and Spal Or67b are consistently separated from the $S$. flava odor response profiles, 630 and Sfla Or67b1 and Sfla Or67b3 are consistently joined, having similar response profiles. 
Figure 5: S. flava Or67b3 confers olfactory sensitivity to ITCs

634 The responses of D. melanogaster flies expressing Dmel Or67b or Sfla Or67b3 under the control 635 of Gal4 in the "empty" neuron (ab3A neuron) and three control parental lines were conducted as

636

637

638

639

640

641

642

643

644

645

646

647

648

649

650

651

652

653

654

655

described in the caption to Figure 1. Genotypes are indicated at the bottom. One arm of the maze was connected to a 1-ml syringe containing a piece of filter paper loaded with $20 \mu 1$ of 1:100 $\mathrm{vol} / \mathrm{vol}$ (gray circles) or 1:1,000 vol/vol (black rectangles) of butyl ITC, while the other arm was connected to a syringe containing a piece of filter paper loaded with $20 \mu \mathrm{l}$ of mineral oil. Data represent the proportion of tests in which insects choose the odorous arm of the maze; the deviation bars indicate the $95 \%$ confidence interval of the proportion. The dotted reference line at 0.5 indicate no preference for either arm of the maze; the numbers between parenthesis indicate the number of tests with choices for one or the other arm. Control flies (first three columns) showed no preference for either arm of the maze, even when tested with the highest concentration of butyl ITC (two-tailed Binomial tests, in all cases $p>0.1$ ). Note that flies carrying the UAS-Sfla Or67b3 transgene under the control of Gal4 in the ab3A neuron were attracted to both concentrations of butyl ITC (two-tailed Binomial tests, $* * * \mathrm{p}<0.005 ; * * * * \mathrm{p}<0.001$ ). In contrast, flies carrying the $U A S$-Dmel Or67b transgene under the control of Gal4 were attracted only to the highest concentration of the odorant (two-tailed Binomial tests, ${ }^{* *} \mathrm{p}<0.01$ ). At the lowest concentration, the response of flies carrying the $S$. flava transgene was different from the response of flies carrying the $D$. melanogaster transgene (Fisher Exact test, $\mathrm{p}<0.05$ ). These results indicate that in these flies, Sfla Or67b3 is sufficient to confer attraction to butyl ITC, a behavior that persists at low concentrations. 
656

657

658

659

660

661

662

663

664

665

666

667

668

669

670

671

672

673

674

675

676

677

\section{Figure 6: A model for the evolution of $O r 67 b$ and comparison of tuning curves of}

orthologous Or22a, Ir75b and Or67b (A) The evolution of Scaptomyza Or67b begins with a shift in the tuning breadth and ligand specificity of an ancestral Or67b, from Dmel activators and GLVs to ITCs (neofunctionalization). Subsequent gene triplication of Sfla Or67b gave rise to three paralogous Or67b genes (Sfla Or67b1, Sfla Or67b2, and Sfla Or67b3). Sfla Or67b1 evolved to become more narrowly tuned, specializing in the detection of a few ITCs

(subfunctionalization), while Sfla Or67b3 kept its ancestral, more broadly tuned state, responding to a broad range of ITCs. The ligands and tuning breadth of Sfla Or67b2 remain unresolved in this model. (B) The ligand specificities of orthologous Or22a, Ir75b, and Or67b. The Or22a orthologs from D. melanogaster (Dmel Or22a), D. sechellia (Dsec Or22a), D. erecta (Der Or22a), and D. suzukii (Dsuz Or22a) are all strongly activated by the host-derived esters indicated (top left; ref. 16, 17, 42, 43). The Ir75b orthologs from D. melanogaster (Dmel Ir75b), and D. sechellia (Dsec Ir75b) are strongly activated by the two acids indicated (top right; ref. 15). Dmel Or67b and Spal Or67b are strongly activated by acetophenone, while Sfla Or67b copies are activated by ITCs only (bottom). Note that Or22a and Ir75b orthologs are both divergent but activated by ligands belonging to a single chemical class (esters and acids, respectively). In contrast, the ligands of orthologous Or67b from Dmel and Spal are responsive to a variety of chemical classes which include alcohols, aldehydes and ketones, whereas Sfla Or67b orthologs are responsive to an entirely different chemical class (ITCs). 


\section{References:}

1. G. Frankel, The raison d'etre of secondary plant substances. Science 129, 1466-1470 (1959).

2. I. Winde, U. Wittstock, Insect herbivore counteradaptations to the plant glucosinolatemyrosinase system. Phytochemistry 72, 1566-1575 (2011).

3. A. D. Gloss, et al., Evolution in an ancient detoxification pathway is coupled with a transition to herbivory in the Drosophilidae. Mol. Biol. Evol. 31, 2441-2456 (2014).

4. A. Kergunteuil, S. Dugravot, A. Mortreuil, A. Le Ralec, A. M. Cortesero, Selecting volatiles to protect brassicaceous crops against the cabbage root fly, Delia radicum. Entomol. Exp. Appl. 144, 69-77 (2012).

5. B. Al-Anzi, W. D. Tracey Jr, S. Benzer, Response of Drosophila to wasabi is mediated by painless, the fly homolog of mammalian TRPA1/ANKTM1. Curr. Biol. 16, 1034-1040 (2006).

6. T. Ohta, T. Imagawa, S. Ito, Novel agonistic action of mustard oil on recombinant and endogenous porcine transient receptor potential V1 (pTRPV1) channels. Biochemical Pharmacology 73, 1646-1656 (2007).

7. L. J. Macpherson, et al., Noxious compounds activate TRPA1 ion channels through covalent modification of cysteines. Nature 445, 541-545 (2007).

8. K. Kang, et al., Analysis of Drosophila TRPA1 reveals an ancient origin for human chemical nociception. Nature 464, 597-600 (2010).

9. O. M. Arenas, et al., Activation of planarian TRPA1 by reactive oxygen species reveals a conserved mechanism for animal nociception. Nat. Neurosci. 20, 1686-1693 (2017).

10. A. Hinman, H.-H. Chuang, D. M. Bautista, D. Julius, TRP channel activation by reversible covalent modification. Proc. Natl. Acad. Sci. U. S. A. 103, 19564-19568 (2006).

11. R. M. Joseph, J. R. Carlson, Drosophila chemoreceptors: A molecular interface between the chemical world and the brain. Trends in Genetics 31, 683-695 (2015).

12. C. Gomez-Diaz, F. Martin, J. M. Garcia-Fernandez, E. Alcorta, The two main olfactory receptor families in Drosophila, ORs and IRs: A Comparative Approach. Front. Cell. Neurosci. 12, 253 (2018).

13. A. Sanchez-Gracia, F. G. Vieira, J. Rozas, "Molecular evolution of the major chemosensory gene families in insects. Heredity (Edinb). 2009; 103 (3): 208-16" (Epub 2009/05/14. doi: 10.1038/hdy. 2009.55. PubMed PMID: 19436326). 
14. P. Brand, S. R. Ramírez, The Evolutionary Dynamics of the Odorant Receptor Gene Family in Corbiculate Bees. Genome Biol. Evol. 9, 2023-2036 (2017).

15. L. L. Prieto-Godino, et al., Evolution of acid-sensing olfactory circuits in drosophilids. Neuron 93, 661-676.e6 (2017).

16. S. Mansourian, et al., Wild African Drosophila melanogaster are seasonal specialists on marula fruit. Curr. Biol. 28, 3960-3968.e3 (2018).

17. J. Linz, et al., Host plant-driven sensory specialization in Drosophila erecta. Proc. Biol. Sci. 280, 20130626 (2013).

18. N. K. Whiteman, et al., Mining the plant-herbivore interface with a leafmining Drosophila of Arabidopsis. Mol. Ecol. 20, 995-1014 (2011).

19. B. Goldman-Huertas, et al., Evolution of herbivory in Drosophilidae linked to loss of behaviors, antennal responses, odorant receptors, and ancestral diet. Proc. Natl. Acad. Sci. U. S. A. 112, 3026-3031 (2015).

20. C. E. Reisenman, Y. Lee, T. Gregory, P. G. Guerenstein, Effects of starvation on the olfactory responses of the blood-sucking bug Rhodnius prolixus. J. Insect Physiol. 59, 717721 (2013).

21. C. P. Faucher, M. Hilker, M. de Bruyne, Interactions of carbon dioxide and food odours in Drosophila: olfactory hedonics and sensory neuron properties. PLoS One 8, e56361 (2013).

22. L. W. Aurand, J. A. Singleton, T. A. Bell, J. L. Etchells, Volatile components in the vapors of natural and distilled vinegars. J. Food Sci. 31, 172-177 (1966).

23. F. J. Cousin, et al., Microorganisms in fermented apple beverages: Current knowledge and future directions. Microorganisms 5 (2017).

24. A. D. Gloss, et al., Evolution of herbivory remodels a Drosophila genome. bioRxiv, 767160 (2019).

25. C. S. McBride, J. R. Arguello, B. C. O’Meara, Five Drosophila genomes reveal nonneutral evolution and the signature of host specialization in the chemoreceptor superfamily. Genetics 177, 1395-1416 (2007).

26. Z. Yang, PAML 4: phylogenetic analysis by maximum likelihood. Mol. Biol. Evol. 24, 1586-1591 (2007).

27. T. S. Ha, D. P. Smith, A pheromone receptor mediates 11-cis-vaccenyl acetate-induced responses in Drosophila. J. Neurosci. 26, 8727-8733 (2006).

28. D. J. Hoare, et al., Modeling peripheral olfactory coding in Drosophila larvae. PLoS One 6, e22996 (2011). 
29. S. G. Chin, S. E. Maguire, P. Huoviala, G. S. X. E. Jefferis, C. J. Potter, Olfactory neurons and brain centers directing oviposition decisions in Drosophila. Cell Rep. 24, 1667-1678 (2018).

30. E. A. Hallem, J. R. Carlson, Coding of odors by a receptor repertoire. Cell 125, 143-160 (2006).

31. F. Gonzalez, P. Witzgall, W. B. Walker, Protocol for heterologous expression of insect odourant receptors in Drosophila. Front. Ecol. Evol. 4, 189 (2016).

32. A. A. Agrawal, N. S. Kurashige, A role for isothiocyanates in plant resistance against the specialist herbivore Pieris rapae. J. Chem. Ecol. 29, 1403-1415 (2003).

33. D. Münch, C. G. Galizia, DoOR 2.0--Comprehensive mapping of Drosophila melanogaster odorant responses. Sci. Rep. 6, 21841 (2016).

34. S. F. Vaughn, M. A. Berhow, Glucosinolate hydrolysis products from various plant sources: $\mathrm{pH}$ effects, isolation, and purification. Industrial Crops and Products 21, 193-202 (2005).

35. P. R. Ehrlich, P. H. Raven, Butterflies and plants: A study in coevolution. Evolution 18, 586-608 (1964).

36. J. A. Depree, T. M. Howard, G. P. Savage, Flavour and pharmaceutical properties of the volatile sulphur compounds of Wasabi (Wasabia japonica). Food Res. Int. 31, 329-337 (1998).

37. E. A. Bernays, Evolution of Feeding Behavior in Insect Herbivores: Success seen as different ways to eat without being eaten. Bioscience 48, 35-44 (1998).

38. S. Ohno, The enormous diversity in genome sizes of fish as a reflection of nature's extensive experiments with gene duplication. Trans. Am. Fish. Soc. 99, 120-130 (1970).

39. H. M. Heidel-Fischer, et al., An insect counteradaptation against host plant defenses evolved through concerted neofunctionalization. Mol. Biol. Evol. 36, 930-941 (2019).

40. X. He, J. Zhang, Rapid subfunctionalization accompanied by prolonged and substantial neofunctionalization in duplicate gene evolution. Genetics 169, 1157-1164 (2005).

41. R. Assis, D. Bachtrog, Neofunctionalization of young duplicate genes in Drosophila. Proc. Natl. Acad. Sci. U. S. A. 110, 17409-17414 (2013).

42. T. Dekker, I. Ibba, K. P. Siju, M. C. Stensmyr, B. S. Hansson, Olfactory shifts parallel superspecialism for toxic fruit in Drosophila melanogaster sibling, D. sechellia. Curr. Biol. 16, 101-109 (2006).

43. I. Keesey, et al., Evolution of a pest: towards the complete neuroethology of Drosophila suzukii and the subgenus Sophophora. BioRxiv (2019). 
44. J. A. A. Renwick, M. Haribal, S. Gouinguené, E. Städler, Isothiocyanates stimulating oviposition by the diamondback moth, Plutella xylostella. J. Chem. Ecol. 32, 755-766 (2006).

45. C. Tait, S. Batra, S. S. Ramaswamy, J. L. Feder, S. B. Olsson, Sensory specificity and speciation: a potential neuronal pathway for host fruit odour discrimination in Rhagoletis pomonella. Proc. Biol. Sci. 283 (2016).

46. J. J. Wiens, R. T. Lapoint, N. K. Whiteman, Herbivory increases diversification across insect clades. Nat. Commun. 6, 8370 (2015).

47. C. Mitter, B. Farrell, B. Wiegmann, The phylogenetic study of adaptive zones: Has phytophagy promoted insect diversification? Am. Nat. 132, 107-128 (1988).

48. J. H. Zar, Biostatistical Analysis (Prentice Hall, 1999).

49. Y. Benjamini, Y. Hochberg, Controlling the false discovery rate: A practical and powerful approach to multiple testing. Journal of the Royal Statistical Society: Series B (Methodological) 57, 289-300 (1995).

50. J. Thurmond, et al., FlyBase 2.0: the next generation. Nucleic Acids Res. 47, D759-D765 (2019).

51. K. Katoh, MAFFT: a novel method for rapid multiple sequence alignment based on fast Fourier transform. Nucleic Acids Research 30, 3059-3066 (2002).

52. L.-T. Nguyen, H. A. Schmidt, A. von Haeseler, B. Q. Minh, IQ-TREE: A fast and effective stochastic algorithm for estimating maximum-likelihood phylogenies. Molecular Biology and Evolution 32, 268-274 (2015).

53. A. Stamatakis, RAxML version 8: a tool for phylogenetic analysis and post-analysis of large phylogenies. Bioinformatics 30, 1312-1313 (2014).

54. F. Ronquist, et al., MrBayes 3.2: efficient Bayesian phylogenetic inference and model choice across a large model space. Syst. Biol. 61, 539-542 (2012).

55. S. Guindon, et al., New Algorithms and methods to estimate maximum-likelihood phylogenies: Assessing the performance of PhyML 3.0. Systematic Biology 59, 307-321 (2010).

56. R. C. Team, R: a language and environment for statistical computing [online]. R Foundation for Statistical Computing, Vienna, Austria (2018).

57. E. Paradis, K. Schliep, ape 5.0: an environment for modern phylogenetics and evolutionary analyses in R. Bioinformatics 35, 526-528 (2019).

58. E. A. Hallem, J. R. Carlson, The odor coding system of Drosophila. Trends in Genetics 20, 453-459 (2004). 
812

813

814

815

816

817

818

819

820

821

822

823

824

825

826

827

828

829

830

831
59. R. T. Lapoint, P. M. O'Grady, N. K. Whiteman, Diversification and dispersal of the Hawaiian Drosophilidae: the evolution of Scaptomyza. Mol. Phylogenet. Evol. 69, 95-108 (2013).

60. J. Bischof, R. K. Maeda, M. Hediger, F. Karch, K. Basler, An optimized transgenesis system for Drosophila using germ-line-specific $\varphi \mathrm{C} 31$ integrases. Proc. Natl. Acad. Sci. U. S. A. 104, 3312-3317 (2007). 
bioRxiv preprint doi: https://doi.org/10.1101/2019.12.27.889774; this version posted December 30, 2019. The copyright holder for this preprint (which was not certified by peer review) is the author/funder, who has granted bioRxiv a license to display the preprint in perpetuity. It is made available under aCC-BY-ND 4.0 International license.

Table S1: Parameters from Odorant Receptor molecular evolution analyses

\begin{tabular}{|c|c|c|c|c|c|}
\hline Test* & Branch $\dagger$ & $\begin{array}{c}\text { background } \\
\mathrm{dN} / \mathrm{dS}\end{array}$ & $\begin{array}{l}\text { foreground } \\
\qquad \mathrm{dN} / \mathrm{dS}\end{array}$ & $\mathrm{dN}-\mathrm{dS}$ & $\begin{array}{c}\text { FDR } \\
\text { adjusted p- } \\
\text { value }\end{array}$ \\
\hline OR & Or2a & 0.10948 & 0.08368 & -1.1523 & 0.4031933 \\
\hline OR & Or10a & 0.16074 & 0.08826 & -0.5837 & 0.03599091 \\
\hline OR & Or13a1 & 0.13815 & 0.95538 & -0.0014 & 8.36204E-06 \\
\hline OR & Or13a2 & 0.14485 & 0.29386 & -0.0114 & 0.5374215 \\
\hline OR & Or13a ancestor & 0.1405 & 0.22696 & -0.2232 & 0.1062494 \\
\hline OR & Or19a & 0.27184 & 0.256 & -0.3699 & 0.8337806 \\
\hline OR & Or22c & 0.15572 & 0.25996 & -0.1496 & 0.1475187 \\
\hline OR & Or23a & 0.16331 & 0.2829 & -0.2438 & 0.0934421 \\
\hline OR & Or24a & 0.08324 & 0.09358 & -0.5121 & 0.7036877 \\
\hline OR & Or30a & 0.07027 & 0.10685 & -0.4864 & 0.1396827 \\
\hline OR & Or33c & 0.20981 & 0.15322 & -0.402 & 0.2748754 \\
\hline OR & Or35a & 0.10058 & 0.80581 & -0.2963 & 0.00245945 \\
\hline OR & Or42a1 & 0.10806 & 0.11791 & -0.336 & 0.7716366 \\
\hline OR & Or42a2 & 0.10578 & 0.80581 & -0.012 & 0.000970903 \\
\hline OR & Or42a3 & 0.10571 & 0.80581 & -0.0045 & 0.000643469 \\
\hline OR & Or $42 \mathrm{a} 2$ and 3 ancestor & 0.11046 & 0.08409 & -0.5505 & 0.3637872 \\
\hline OR & Or43a & 0.14775 & 0.08518 & -0.3217 & 0.1557934 \\
\hline OR & Or45b & 0.10067 & 0.10449 & -0.3992 & 0.8803498 \\
\hline OR & Or46a & 0.14313 & 0.20832 & -0.2632 & 0.1151226 \\
\hline OR & Or47b & 0.16957 & 0.15781 & -0.3375 & 0.8025064 \\
\hline OR & Or49a & 0.19774 & 0.17914 & -0.5377 & 0.7036877 \\
\hline OR & Or49b & 0.10784 & 0.05836 & -0.3747 & 0.09592573 \\
\hline OR & Or56a & 0.12598 & 0.05652 & -0.5602 & $\mathbf{0 . 0 0 4 2 9 8 5 1 7}$ \\
\hline OR & Or59a & 0.1085 & 0.31529 & -0.2475 & 0.000415309 \\
\hline OR & Or59b & 0.09936 & 0.23227 & -0.4421 & 0.000415309 \\
\hline OR & Or63a1 & 0.12346 & $\infty$ & 0.0172 & 3.51892E-05 \\
\hline OR & Or63a2 & 0.12136 & $\infty$ & 0.0274 & 1.35815E-09 \\
\hline OR & Or63a ancestor & 0.10903 & 0.56602 & -0.1212 & $5.0862 E-13$ \\
\hline OR & Or67a1 & 0.20346 & 0.13116 & -0.528 & 0.0934421 \\
\hline OR & Or67a2 & 0.2067 & 0.06369 & -0.0693 & 0.0004729 \\
\hline OR & Or67b1 & 0.11719 & 0.87005 & -0.0059 & 2.5914E-07 \\
\hline
\end{tabular}


bioRxiv preprint doi: https://doi.org/10.1101/2019.12.27.889774; this version posted December 30, 2019. The copyright holder for this preprint (which was not certified by peer review) is the author/funder, who has granted bioRxiv a license to display the preprint in perpetuity. It is made available under aCC-BY-ND 4.0 International license.

\begin{tabular}{|c|c|c|c|c|c|}
\hline OR & Or67b2 & 0.11479 & 0.69462 & -0.0253 & 1.96849E-09 \\
\hline OR & Or67b3 & 0.10844 & 1.95161 & 0.0414 & $9.78045 E-20$ \\
\hline OR & Or67b1 and 2 ancestor & 0.11932 & $\infty$ & $\mathbf{0 . 0 2 8 2}$ & 3.90702E-08 \\
\hline OR & Or67b1, 2 and 3 ancestor & 0.11679 & 0.28275 & -0.2591 & 0.000585544 \\
\hline OR & Or67c & 0.05491 & 0.07255 & -0.4886 & 0.4029281 \\
\hline OR & Or67d & 0.12277 & 7.22359 & 0.0948 & 2.56153E-05 \\
\hline OR & Or69a & 0.21665 & 0.12519 & -0.4274 & 0.05279388 \\
\hline OR & Or71a & 0.15846 & 0.25285 & -0.3464 & 0.1151226 \\
\hline OR & Or74a1 & 0.13897 & 0.16825 & -0.3766 & 0.5728869 \\
\hline OR & Or74a2 & 0.13723 & 0.18825 & -0.4375 & 0.2932306 \\
\hline OR & Or82a & 0.12569 & 0.18201 & -0.2805 & 0.1926929 \\
\hline OR & Or83a & 0.0967 & 0.13983 & -0.4518 & 0.148133 \\
\hline OR & Or83c1 & 0.16267 & 0.21756 & -0.4381 & 0.3145556 \\
\hline OR & Or83c2 & 0.22615 & 0.41682 & -0.1561 & 0.0934421 \\
\hline OR & Or83c3 & 0.22488 & 0.39035 & -0.2043 & 0.0934421 \\
\hline OR & Or83c 2 and 3 ancestor & 0.25561 & 0.17609 & -0.2679 & 0.5410598 \\
\hline OR & Or85aLike & 0.22488 & 0.39035 & -1.8882 & 0.001826812 \\
\hline OR & Or85bc & 0.19387 & 0.07261 & -0.32 & 0.2783313 \\
\hline OR & Or85e & 0.15034 & 0.20391 & -0.3561 & 0.6592839 \\
\hline OR & Or85f & 0.11206 & 0.13076 & -0.4139 & 0.08611496 \\
\hline OR & Or88a & 0.18031 & 0.09816 & -0.2848 & 0.003678095 \\
\hline OR & Or92a & 0.14147 & 0.32458 & -0.3894 & 3.51892E-05 \\
\hline OR & Or94a & 0.044 & 0.13816 & -0.4616 & 0.1933 \\
\hline OR & Or94b & 0.09284 & 0.14353 & -0.5473 & 0.09592573 \\
\hline OR & Or98a1 & 0.09893 & 0.17168 & 0.0013 & 0.1796849 \\
\hline OR & Or98a3 & 0.18051 & 1.69406 & -0.0093 & 0.6770641 \\
\hline OR & Or98a7 & 0.18219 & 0.28105 & 0.0258 & 5.47517E-05 \\
\hline OR & Or98a1 and 3 ancestor & 0.14909 & 2.74217 & -0.0113 & 0.00014207 \\
\hline OR & Or98a1, 3 and 7 ancestor & 0.15018 & 0.81658 & -0.6623 & 0.7716366 \\
\hline OR & Or98a2 & 0.18749 & 0.17369 & -0.024 & 0.7716366 \\
\hline OR & Or98a6 & 0.24649 & 0.30314 & -0.044 & 0.6592839 \\
\hline OR & Or98a 2 and 6 ancestor & 0.24491 & 0.30931 & -0.33 & 0.0934421 \\
\hline OR & Or98a4 & 0.2739 & 0.14173 & 0.0063 & 0.000676304 \\
\hline OR & Or98a5 & 0.22865 & 1.23866 & 0.01 & 0.05279388 \\
\hline OR & Or98a4 and 5 ancestor & 0.237 & 4.11669 & -0.4823 & 0.01935304 \\
\hline
\end{tabular}


bioRxiv preprint doi: https://doi.org/10.1101/2019.12.27.889774; this version posted December 30, 2019. The copyright holder for this preprint (which was not certified by peer review) is the author/funder, who has granted bioRxiv a license to display the preprint in perpetuity. It is made available under aCC-BY-ND 4.0 International license.

\begin{tabular}{|c|c|c|c|c|c|}
\hline OR & Or98b1 & 0.28857 & 0.13007 & -0.8492 & 0.7613693 \\
\hline OR & Or98bLike & 0.18605 & 0.20625 & -0.8656 & 0.5728869 \\
\hline OR & OrN1 & 0.19137 & 0.15525 & -0.3194 & 0.1960608 \\
\hline OR & OrN2 copy 1 & 0.02988 & 0.07861 & -0.0006 & 0.1278416 \\
\hline OR & OrN2 copy 4 & 0.14809 & 0.21155 & 0.0008 & 0.05293525 \\
\hline OR & OrN2 copy 5 & 0.2495 & 0.94941 & -0.6183 & 0.08611496 \\
\hline OR & OrN2 copy 6 & 0.24826 & 1.05198 & -0.8924 & 0.1151226 \\
\hline OR & OrN 2 copy 1 and 4 ancestor & 0.26274 & 0.16626 & -0.4861 & 0.5728869 \\
\hline OR & Orco & 0.26137 & 0.17749 & -0.2037 & 0.02049901 \\
\hline OR67b & D. grimshawi & 0.24934 & 0.29184 & -0.3355 & 0.7873258 \\
\hline OR67b & S. flava copy 3 & 0.1041 & 1.803 & $\mathbf{0 . 0 3 7 5}$ & $1.44394 \mathrm{E}-21$ \\
\hline OR67b & S. flava copy 2 & 0.10935 & 0.7393 & -0.0208 & 1.05769E-10 \\
\hline OR67b & S. flava copy 1 & 0.11251 & 0.62856 & -0.022 & $1.5606 \mathrm{E}-06$ \\
\hline OR67b & S. pallida & 0.1236 & 0.0506 & -0.3076 & 0.01604471 \\
\hline OR67b & D. mojavensis & 0.1137 & 0.2053 & -0.8484 & 0.009320281 \\
\hline OR67b & D. virilis & 0.119 & 0.1185 & -1.4528 & 0.9933245 \\
\hline OR67b & D. melanogaster & 0.11971 & 0.04714 & -0.0423 & 0.4999046 \\
\hline OR67b & D. sechellia & 0.1193 & 0.0796 & -0.0368 & 0.6982622 \\
\hline OR67b & D. simulans & 0.1202 & 0.0197 & -0.0523 & 0.04609565 \\
\hline OR67b & D. yakuba & 0.1198 & 0.0914 & -0.1247 & 0.614736 \\
\hline OR67b & D. erecta & 0.1193 & 0.1051 & -0.1126 & 0.7873258 \\
\hline OR67b & D. ananassae & 0.1329 & 0.0303 & -1.7359 & $5.2654 \mathrm{E}-10$ \\
\hline OR67b & D. persimilis & 0.1188 & 0.1803 & -0.0103 & 0.7873258 \\
\hline OR67b & D. pseudoobscura & 0.119 & 0.1149 & -0.0078 & 0.9933245 \\
\hline OR67b & D. willistoni & 0.1288 & 0.0427 & -1.4866 & $1.97605 \mathrm{E}-05$ \\
\hline OR67b & Subgenus Drosophila Ancestor & 0.1236 & 0.0564 & -0.9778 & 0.01967709 \\
\hline OR67b & Sophophora minus D. willistoni & 0.1212 & 0.0711 & -0.5393 & 0.3034497 \\
\hline OR67b & virilis-repleta radiation & 0.1196 & 0.0905 & -0.1587 & 0.7873258 \\
\hline OR67b & Hawaiian radiation ancestor & 0.1198 & 0.0979 & -0.2595 & 0.7817405 \\
\hline OR67b & Scaptomyza genus ancestor & 0.1221 & 0.0435 & -0.2512 & 0.04498955 \\
\hline OR67b & S. flava copy 1,2 and 3 ancestor & 0.1094 & 0.4081 & -0.1282 & 4.06804E-07 \\
\hline OR67b & S. flava copy 1 and 2 ancestor & 0.1135 & $\infty$ & 0.0276 & 4.5389E-09 \\
\hline OR67b & D. simulans and D. sechelia ancestor & 0.1205 & 0.0001 & -0.0564 & 0.01202915 \\
\hline OR67b & D. yakuba and D. erecta ancestor & 0.1221 & 0.0196 & -0.1444 & 0.00128517 \\
\hline OR67b & melanogaster subgroup minus Dmel & 0.1201 & 0.048 & -0.0727 & 0.2412147 \\
\hline
\end{tabular}


bioRxiv preprint doi: https://doi.org/10.1101/2019.12.27.889774; this version posted December 30, 2019. The copyright holder for this preprint (which was not certified by peer review) is the author/funder, who has granted bioRxiv a license to display the preprint in perpetuity. It is made available under aCC-BY-ND 4.0 International license.

\begin{tabular}{|l|l|r|r|r|r|}
\hline OR67b & melanogaster subgroup ancestor & 0.125 & 0.0446 & -0.5921 & $\mathbf{0 . 0 0 3 3 4 8 6 1 6}$ \\
\hline OR67b & melanogaster group ancestor & 0.1207 & 0.0634 & -0.2523 & 0.4817083 \\
\hline OR67b & pseudoobscura subgroup ancestor & 0.1291 & 0.0342 & -0.9693 & $\mathbf{2 . 3 9 7 6 6 E - 0 6}$ \\
\hline
\end{tabular}

Table S1: Parameters for background versus foreground branch tests in PAML. ${ }^{*} \mathrm{OR}=\mathrm{Branch}$ tests for differences in $\mathrm{dN} / \mathrm{dS}$ rates among Scaptomyza flava copies of odorant receptor genes. $\mathrm{OR} 67 \mathrm{~b}=$ Branch tests for differences in $\mathrm{dN} / \mathrm{dS}$ rates among all individual branches in a phylogeny of Or67b homologs in Scaptomyza and Drosophila spp. $\uparrow$ Terminal branches are indicated with gene names. Ancestral branches are indicated with the term "ancestor" and a list of the terminal branches or the clade subtended.

TableS2 PCR Primers

\begin{tabular}{|l|l|l|}
\hline \multicolumn{1}{|c|}{ Primer Name } & \multicolumn{1}{|c|}{ Sequence } & \multicolumn{1}{c|}{ Primer role } \\
\hline 67b12Fb & cATGAAGGACTTATTGGATCTGGAGCTAG & CDS amplification \\
\hline $67 \mathrm{~b} 1 \mathrm{Rb}$ & agcgcaTTATTTGTCTCTCATATTGCTC & CDS amplification \\
\hline 67b2Rb & agcgcaTTATTCATCTCTCATGTTACGC & CDS amplification \\
\hline 67b3Fb & cATGAACTTATTGGATATGGAGCTAG & CDS amplification \\
\hline 67b3Rb & agcgcaTTATTTGTTAGATAAATTACGC & CDS amplification \\
\hline Sp67bFb & cATGAAAAACCTATTGGACCGGAAG & CDS amplification \\
\hline Sp67bRb & aaagtacaTCATTTGTTATTCATATTACGCAAAAG & CDS amplification \\
\hline b1EcoRIF & GACgaattcATGAAGGACTTATTGG & RE cut site addition \\
\hline b1KpnIR & GACggtaccTTATTTGTCTCTCATATTG & RE cut site addition \\
\hline b2KpnIR & GACggtaccTTATTCATCTCTCATG & RE cut site addition \\
\hline b3KpnIR & GACggtaccTTATTTGTTAGATAAATTACG & RE cut site addition \\
\hline PalEcoRIF & GACgaattcATGAAAAACCTATTGGACC & RE cut site addition \\
\hline PalKpnIR & GACggtaccTCATTTGTTATTCATATTACG & RE cut site addition \\
\hline
\end{tabular}

Tables S2: Nucleotides in lower case are either in untranslated sequence (CDS amplification) or are restriction enzyme cut sites (RE cut site addition). CDS amplification primers were used to amplify full Or67b CDS sequence from cDNA. Primers labeled "RE cut site addition" were used to engineer restriction enzyme cut-sites via PCR mutagenesis in order to ligate Or67b CDS sequences into the pUASTattB plasmid. All sequences are listed in a 5' to 3' orientation. 
bioRxiv preprint doi: https://doi.org/10.1101/2019.12.27.889774; this version posted December 30, 2019. The copyright holder for this preprint (which was not certified by peer review) is the author/funder, who has granted bioRxiv a license to display the preprint in perpetuity. It is made available under aCC-BY-ND 4.0 International license.

\begin{tabular}{|c|cc|cc|}
\hline \multicolumn{2}{|c|}{ Table S3: Net number of spikes/second; average \pm SE } & \\
\hline Odors & "Empty neuron" & \multicolumn{2}{|c|}{ WT Or22a } & n \\
\hline ethyl hexanoate & spikes & $\mathbf{n}$ & $118 \pm 7.7$ & 7 \\
mineral oil & 0 & 7 & $6.33 \pm 1.7$ & 6 \\
\hline acetophenone & 0 & 7 & NA & NA \\
butyl ITC & 0 & 6 & NA & NA \\
mandelonitrile & 0 & 6 & NA & NA \\
cis-3-hexenyl & 0 & 6 & NA & NA \\
butyrate & 0 & 6 & NA & NA \\
trans-2-pentenal & 0 & 6 & NA & NA \\
geranyl acetate & 0 & 6 & NA \\
mineral oil & 0 & 7 & & \\
\hline
\end{tabular}

\begin{tabular}{|c|c|c|c|c|c|c|c|c|c|c|}
\hline \multirow[b]{2}{*}{ Odors } & \multicolumn{2}{|c|}{ Dmel67b } & \multicolumn{2}{|c|}{ Spal67b } & \multicolumn{2}{|c|}{ Sfla67b1 } & \multicolumn{2}{|l|}{ Sfla67b2 } & \multicolumn{2}{|l|}{ Sfla67b3 } \\
\hline & spikes & $\mathbf{n}$ & spikes & $\mathbf{n}$ & spikes & $\mathbf{n}$ & spikes & $\mathbf{n}$ & spikes & $\mathbf{n}$ \\
\hline apple cider vinegar & $96.3 \pm 8.0$ & 6 & $62 \pm 10.4$ & 7 & $-4.33 \pm 2.0$ & 6 & $6.67 \pm 4.6$ & 6 & $15 \pm 2.1$ & 6 \\
\hline arugula & $19.7 \pm 3.0$ & 6 & $14 \pm 3.4$ & 7 & $64 \pm 7.8$ & 6 & $24 \pm 8.7$ & 6 & $22.3 \pm 2.3$ & 6 \\
\hline wasabi & $2.67 \pm 2.0$ & 6 & $2.67 \pm 1.11$ & 6 & $15.3 \pm 1.9$ & 8 & $16.2 \pm 2.5$ & 8 & $13.7 \pm 4.4$ & 6 \\
\hline horseradish & $3.67 \pm 1.8$ & 6 & $-0.33 \pm 1.0$ & 6 & $8.75 \pm 1.3$ & 8 & $12.9 \pm 2.0$ & 8 & $31.7 \pm 4.4$ & 6 \\
\hline turnip & $1 \pm 1.8$ & 6 & $0.67 \pm 0.7$ & 6 & $11.8 \pm 1.6$ & 8 & $22 \pm 2.7$ & 8 & $34.3 \pm 1.4$ & 6 \\
\hline daikon & $2 \pm 3.2$ & 6 & $-0.67 \pm 0.8$ & 6 & $14 \pm 2.4$ & 8 & $17.1 \pm 3.4$ & 8 & $19 \pm 3.4$ & 6 \\
\hline beet & $8 \pm 3.8$ & 6 & $-0.33 \pm 1.1$ & 6 & $2 \pm 1.30$ & 6 & $-0.33 \pm 1.7$ & 6 & $4.33 \pm 2.2$ & 6 \\
\hline \multicolumn{11}{|l|}{$\begin{array}{l}\text { Dmel67b } \\
\text { activators }\end{array}$} \\
\hline acetophenone & $176 \pm 18$ & 7 & $78 \pm 2.4$ & 20 & $3.27 \pm 1.2$ & 11 & $4.5 \pm 2.0$ & 8 & $1.57 \pm 1.3$ & 14 \\
\hline cis-3-hexen-1-ol & $56.9 \pm 20$ & 7 & $22 \pm 4.1$ & 7 & $8.67 \pm 2.0$ & 6 & $6.75 \pm 1.3$ & 8 & $2.67 \pm 2.3$ & 6 \\
\hline $\begin{array}{c}4- \\
\text { methylcyclohexenol }\end{array}$ & $80.7 \pm 8.7$ & 6 & $33 \pm 4.4$ & 13 & $8.89 \pm 4.3$ & 9 & $1.56 \pm 1.4$ & 9 & $2.67 \pm 1.2$ & 6 \\
\hline phenethyl alcohol & $140 \pm 19$ & 8 & $26.3 \pm 4.5$ & 6 & $2.67 \pm 1.2$ & 6 & $1 \pm 1.2$ & 6 & $3.67 \pm 1.5$ & 6 \\
\hline 1-hexanol & $118 \pm 13$ & 7 & 19.142857 & 7 & $13.7 \pm 3.8$ & 6 & $10.4 \pm 4.6$ & 9 & $8 \pm 1.9$ & 6 \\
\hline $\begin{array}{l}\text { ethyl (S)-(+)-3- } \\
\text { hydroxybutanoate }\end{array}$ & $15.7 \pm 3.3$ & 7 & $12 \pm 2.8$ & 7 & $4.33 \pm 4.4$ & 6 & $10.4 \pm 2.9$ & 9 & $8 \pm 2$ & 6 \\
\hline 2-hexanol & $12.3 \pm 5.2$ & 8 & $11.7 \pm 2.8$ & 7 & $7.33 \pm 2.1$ & 6 & $9.5 \pm 1.8$ & 8 & $7 \pm 3.1$ & 6 \\
\hline benzyl alcohol & $48.6 \pm 2.9$ & 6 & $14.3 \pm 3.7$ & 7 & $-1.33 \pm 0.8$ & 6 & $6.75 \pm 1.8$ & 8 & $5.67 \pm 1.4$ & 6 \\
\hline
\end{tabular}


bioRxiv preprint doi: https://doi.org/10.1101/2019.12.27.889774; this version posted December 30, 2019. The copyright holder for this preprint (which was not certified by peer review) is the author/funder, who has granted bioRxiv a license to display the preprint in perpetuity. It is made available under aCC-BY-ND 4.0 International license.

\begin{tabular}{|c|c|c|c|c|c|c|c|c|c|c|}
\hline 2-hexanone & $40 \pm 16$ & 7 & $7.14 \pm 2.0$ & 7 & $9.33 \pm 4.8$ & 6 & $7.5 \pm 2.0$ & 8 & $7.33 \pm 3.5$ & 6 \\
\hline hexyl-formate & $39.3 \pm 8.7$ & 6 & $16.3 \pm 5.6$ & 7 & $7 \pm 2.0$ & 6 & $8.89 \pm 4.5$ & 9 & $3 \pm 0.9$ & 6 \\
\hline phenethyl aldehyde & $51.4 \pm 10$ & 7 & $29.4 \pm 3.8$ & 7 & $2 \pm 2.3$ & 6 & $2.86 \pm 3.0$ & 7 & $1 \pm 0.7$ & 6 \\
\hline 2-heptanone & $28 \pm 4.1$ & 7 & $13.1 \pm 2.3$ & 7 & $6.33 \pm 2.4$ & 6 & $15.3 \pm 2.5$ & 9 & $6 \pm 1.9$ & 7 \\
\hline \multicolumn{11}{|l|}{ ITCs } \\
\hline butyl ITC & $4.57 \pm 1.6$ & 7 & $15 \pm 4.2$ & 6 & $196 \pm 15$ & 7 & $22.7 \pm 2.8$ & 6 & $171 \pm 11$ & 7 \\
\hline ethyl ITC & $0.33 \pm 1.5$ & 6 & $2.33 \pm 0.95$ & 6 & $24 \pm 2.7$ & 6 & $3.33 \pm 3.9$ & 6 & $13 \pm 1.7$ & 6 \\
\hline isobutyl ITC & $1.67 \pm 2.4$ & 6 & $5.33 \pm 2.6$ & 6 & $74 \pm 12$ & 7 & $4.33 \pm 1.8$ & 6 & $139 \pm 17$ & 7 \\
\hline sec-butyl ITC & $4.29 \pm 1.0$ & 7 & $5 \pm 1.8$ & 6 & $9.14 \pm 2.7$ & 7 & $5.33 \pm 2.3$ & 6 & $121 \pm 17$ & 8 \\
\hline allyl ITC & $3.43 \pm 0.8$ & 7 & $9.75 \pm 4.2$ & 8 & $28.9 \pm 5.3$ & 7 & $6 \pm 2.4$ & 7 & $4.75 \pm 3.7$ & 8 \\
\hline $\begin{array}{c}\text { 3-methylthio propyl } \\
\text { ITC }\end{array}$ & $2 \pm 1.8$ & 6 & $6.33 \pm 2.2$ & 6 & $179 \pm 9.2$ & 6 & $33 \pm 6.0$ & 6 & $137 \pm 17$ & 8 \\
\hline benzyl ITC & $2.29 \pm 1.4$ & 7 & $3.75 \pm 1.9$ & 8 & $81.7 \pm 14.4$ & 7 & $3.14 \pm 1.3$ & 7 & $134 \pm 14$ & 8 \\
\hline phenethyl ITC & $6.57 \pm 2.2$ & 7 & $5.25 \pm 2.8$ & 8 & $4.8 \pm 1.3$ & 10 & $1.14 \pm 0.86$ & 7 & $158 \pm 15$ & 8 \\
\hline $\begin{array}{c}\text { 3-methoxybenzyl } \\
\text { ITC }\end{array}$ & $\begin{array}{c}- \\
0.67 \pm 1.1\end{array}$ & 6 & $7 \pm 3.3$ & 6 & $4.57 \pm 2.2$ & 7 & $1.67 \pm 0.80$ & 6 & $3.14 \pm 2.1$ & 7 \\
\hline \multicolumn{11}{|l|}{ Nitriles } \\
\hline mandelonitrile & $45.3 \pm 11$ & 6 & $1 \pm 1$ & 6 & $1.33 \pm 1.8$ & 6 & $-0.29 \pm 0.7$ & 7 & $4 \pm 3.0$ & 6 \\
\hline adiponitrile & $2.67 \pm 2.5$ & 6 & $0.33 \pm 0.33$ & 6 & $0.33 \pm 0.3$ & 6 & $0.67 \pm 2.2$ & 6 & $4.33 \pm 1.8$ & 6 \\
\hline propionitrile & $5.33 \pm 1.6$ & 6 & $1.33 \pm 0.4$ & 6 & $2.3 \pm 2.0$ & 6 & $1.33 \pm 1.1$ & 6 & $4.33 \pm 2.5$ & 6 \\
\hline \multicolumn{11}{|l|}{ GLVs } \\
\hline $\begin{array}{l}\text { cis-3-hexenyl } \\
\text { butyrate }\end{array}$ & $91 \pm 21$ & 7 & $49.3 \pm 8.0$ & 6 & $5 \pm 2.6$ & 8 & $2 \pm 1.8$ & 6 & $11.8 \pm 2.5$ & 8 \\
\hline trans-2-hexen-1-ol & $33 \pm 6.0$ & 6 & $51 \pm 11$ & 6 & $23.5 \pm 5.6$ & 8 & $3 \pm 2.5$ & 6 & $6.29 \pm 2.2$ & 7 \\
\hline $\begin{array}{l}\text { trans-2-hexenyl } \\
\text { acetate }\end{array}$ & $55.7 \pm 19$ & 7 & $34 \pm 6.8$ & 6 & $2 \pm 2.1$ & 6 & $17.3 \pm 2.5$ & 6 & $10.3 \pm 1.9$ & 8 \\
\hline $\begin{array}{l}\text { trans-2-hexenyl } \\
\text { butyrate }\end{array}$ & $29.7 \pm 6.7$ & 6 & $35.6 \pm 8.2$ & 6 & $1.71 \pm 1.0$ & 7 & $4 \pm 1.5$ & 6 & $12 \pm 4.3$ & 7 \\
\hline $\begin{array}{l}\text { cis- }- \text {-hexenyi } \\
\text { acetate }\end{array}$ & $43.3 \pm 9.3$ & 6 & $13.7 \pm 2.6$ & 6 & $1.67 \pm 1.7$ & 6 & $16 \pm 5.3$ & 8 & $11 \pm 5.8$ & 6 \\
\hline \multicolumn{11}{|l|}{$\begin{array}{l}\text { TrpA1 activators/ } \\
\text { electrophiles }\end{array}$} \\
\hline trans-2-pentenal & $27.1 \pm 20$ & 7 & $6.33 \pm 0.8$ & 6 & $4.33 \pm 2.9$ & 6 & $4 \pm 1.4$ & 6 & $4 \pm 1.3$ & 6 \\
\hline citronellal & $1.43 \pm 1.6$ & 7 & $7 \pm 2.3$ & 6 & $4.57 \pm 1.6$ & 7 & $9.14 \pm 3.5$ & 7 & $12 \pm 2.8$ & 11 \\
\hline cinnamaldehyde & $12.3 \pm 2.8$ & 7 & $9 \pm 3.8$ & 6 & $9.25 \pm 2.7$ & 8 & $3 \pm 1$ & 6 & $6 \pm 1.7$ & 12 \\
\hline
\end{tabular}


bioRxiv preprint doi: https://doi.org/10.1101/2019.12.27.889774; this version posted December 30, 2019. The copyright holder for this preprint (which was not certified by peer review) is the author/funder, who has granted bioRxiv a license to display the preprint in perpetuity. It is made available under aCC-BY-ND 4.0 International license.

\begin{tabular}{|c|cc|cc|cc|cc|cc|} 
acrorelin & $2.67 \pm 2.6$ & 6 & $6.33 \pm 2.2$ & 6 & $6.67 \pm 1.9$ & 6 & $7 \pm 4.3$ & 6 & $3.33 \pm 1.3$ & 6 \\
iodoacetamide & $1.43 \pm 1.4$ & 7 & $6 \pm 1.9$ & 6 & $7.33 \pm 1.6$ & 6 & $6 \pm 2.4$ & 6 & $4 \pm 1.7$ & 6 \\
$\begin{array}{c}\text { formaldehyde } \\
\text { N-methylmaleimide }\end{array}$ & $3.14 \pm 2.0$ & 7 & $5.33 \pm 1.8$ & 6 & $6 \pm 2.9$ & 6 & $3 \pm 1.4$ & 6 & $2 \pm 1.0$ & 6 \\
N- & $1.71 \pm 1.0$ & 7 & $8.67 \pm 2.6$ & 6 & $7.33 \pm 1.8$ & 6 & $10 \pm 1.9$ & 6 & $3 \pm 1.3$ & 6 \\
hydroxysuccinimide & & 7 & $4.67 \pm 2.5$ & 6 & $4.67 \pm 2.6$ & 6 & $8.67 \pm 2.3$ & 6 & $2 \pm 0.7$ & 6 \\
methyl salicylate & $-3 \pm 1.8$ & 7 & $0 \pm 0.5$ & 6 & $3 \pm 3$ & 6 & $-0.57 \pm 1.8$ & 7 & $5.33 \pm 4.2$ & 6 \\
S-allyl 2-propene-1- & $5.66 \pm 1.0$ & 7 & $-0.33 \pm 0.8$ & 6 & $3.67 \pm 1.5$ & 6 & $0.57 \pm 1.1$ & 7 & $3 \pm 1.5$ & 6 \\
sulfinothioate & & & & & & & & & & \\
diallyl disulfide & $3.33 \pm 3.3$ & 7 & $0.67 \pm 0.8$ & 6 & $1.33 \pm 0.7$ & 6 & $1.14 \pm 0.6$ & 7 & $3.67 \pm 2.1$ & 6 \\
\hline Others & & & & & & & & & & \\
\hline geranyl acetate & $5.67 \pm 1.0$ & 6 & $26 \pm 6.5$ & 6 & $8.5 \pm 2.4$ & 8 & $4.67 \pm 2.0$ & 6 & $5.25 \pm 1.4$ & 8 \\
limonene & $2.29 \pm 2.4$ & 7 & $5 \pm 4.1$ & 6 & $4.33 \pm 1.6$ & 6 & $1 \pm 0.7$ & 6 & $2.71 \pm 1.5$ & 14 \\
indol-3-carbinol & $4 \pm 1.5$ & 6 & $3.33 \pm 2.6$ & 6 & $5.67 \pm 1.6$ & 6 & $8.67 \pm 1.1$ & 6 & $3.33 \pm 0.8$ & 6 \\
benzyl thyocyanate & $2.57 \pm 1.4$ & 7 & $7.67 \pm 1.4$ & 6 & $8.67 \pm 2.4$ & 6 & $5 \pm 1.3$ & 6 & $8.67 \pm 2.8$ & 6 \\
\hline Solvent control & & & & & & & & & & \\
\hline $\begin{array}{c}\text { DMSO } \\
\text { mineral oil }\end{array}$ & $2.86 \pm 1.6$ & 7 & $10.7 \pm 3.2$ & 6 & $3.33 \pm 1.0$ & 6 & $7.67 \pm 1.4$ & 6 & $4.33 \pm 2.9$ & 6 \\
\hline
\end{tabular}

\begin{tabular}{|c|c|c|c|c|}
\hline \multirow[b]{2}{*}{ odorants } & \multicolumn{2}{|c|}{ Sfla67b1 } & \multicolumn{2}{|c|}{ Sfla67b3 } \\
\hline & spikes & $\mathbf{n}$ & spikes & $\mathbf{n}$ \\
\hline \multirow{2}{*}{\multicolumn{5}{|c|}{$\begin{array}{c}\text { 3-methylthio propyl } \\
\text { ITC }\end{array}$}} \\
\hline & & & & \\
\hline $1 / 100$ & $114 \pm 18$ & 8 & $127 \pm 13$ & 6 \\
\hline $1 / 1,000$ & $64 \pm 11$ & 8 & $27.7 \pm 7.1$ & 6 \\
\hline $1 / 10,000$ & $27.9 \pm 4.6$ & 8 & $11.7 \pm 5.3$ & 6 \\
\hline $1 / 100,000$ & $12 \pm 3.3$ & 9 & $10 \pm 4.0$ & 6 \\
\hline \multicolumn{5}{|l|}{ butyl ITC } \\
\hline $1 / 100$ & $147 \pm 19$ & 10 & $161 \pm 16$ & 6 \\
\hline $1 / 1,000$ & $46.8 \pm 6.6$ & 9 & $50.7 \pm 8.1$ & 6 \\
\hline $1 / 10,000$ & $25.6 \pm 7.3$ & 8 & $19.7 \pm 6.2$ & 6 \\
\hline $1 / 100,000$ & $14.25 \pm 5$ & 9 & $7.33 \pm 3.4$ & 6 \\
\hline isobutyl ITC & & & & \\
\hline
\end{tabular}


bioRxiv preprint doi: https://doi.org/10.1101/2019.12.27.889774; this version posted December 30, 2019. The copyright holder for this preprint (which was not certified by peer review) is the author/funder, who has granted bioRxiv a license to display the preprint in perpetuity. It is made available under aCC-BY-ND 4.0 International license.

\begin{tabular}{|c|c|c|c|c|}
\hline $1 / 100$ & $40.6 \pm 9.0$ & 8 & $131 \pm 14$ & 6 \\
\hline $1 / 1,000$ & $6.3 \pm 2.3$ & 8 & $31.7 \pm 5.6$ & 6 \\
\hline $1 / 10,000$ & $5.63 \pm 2.7$ & 8 & $9 \pm 3.8$ & 6 \\
\hline $1 / 100,000$ & $8.25 \pm 1.8$ & 9 & $6 \pm 5.2$ & 6 \\
\hline \multicolumn{5}{|l|}{ sec-butyl ITC } \\
\hline $1 / 100$ & $5 \pm 1.7$ & 9 & $124 \pm 13$ & 6 \\
\hline $1 / 1,000$ & $4.5 \pm 1.6$ & 9 & $26.3 \pm 4.2$ & 6 \\
\hline $1 / 10,000$ & $5.63 \pm 1.8$ & 8 & $13.7 \pm 4.6$ & 6 \\
\hline $1 / 100,000$ & $10.5 \pm 3.5$ & 8 & $9 \pm 4.5$ & 6 \\
\hline \multicolumn{5}{|l|}{ benzyl ITC } \\
\hline $1 / 100$ & $94.5 \pm 14.1$ & 10 & $123 \pm 13$ & 6 \\
\hline $1 / 1,000$ & $16.5 \pm 5.1$ & 9 & $27.7 \pm 9.4$ & 6 \\
\hline $1 / 10,000$ & $8.625 \pm 3.6$ & 8 & $8 \pm 1.4$ & 6 \\
\hline $1 / 100,000$ & $10.5 \pm 3.2$ & 9 & $13 \pm 5.0$ & 6 \\
\hline \multicolumn{5}{|l|}{ phenethyl ITC } \\
\hline $1 / 100$ & $5.25 \pm 2.8$ & 9 & $147 \pm 17$ & 6 \\
\hline $1 / 1,000$ & $8.5 \pm 3.0$ & 8 & $74 \pm 18$ & 6 \\
\hline $1 / 10,000$ & $8.71 \pm 4.1$ & 7 & $26.3 \pm 7.4$ & 6 \\
\hline $1 / 100,000$ & $8 \pm 2.9$ & 7 & $9.33 \pm 5.2$ & 6 \\
\hline mineral oil & $1 \pm 1.4$ & 6 & $0 \pm 0.4$ & 11 \\
\hline
\end{tabular}

Tables S3: Responses (net number of spikes/second; average \pm SE) of the ab3 sensilla of flies expressing either no Or22a ("Empty neuron"), wild-type Or22a (Canton-S), Dmel Or67b, Spal Or67b, Sfla Or67b1, Sfla Or67b2 or Or67b3 to stimulation with the odors and number of recordings (n) are denoted. 
bioRxiv preprint doi: https://doi.org/10.1101/2019.12.27.889774; this version posted December 30, 2019. The कopyiright holderfor (tils preprint

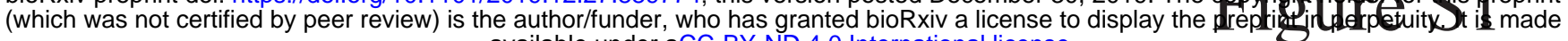
available under aCC-BY-ND 4.0 International license.

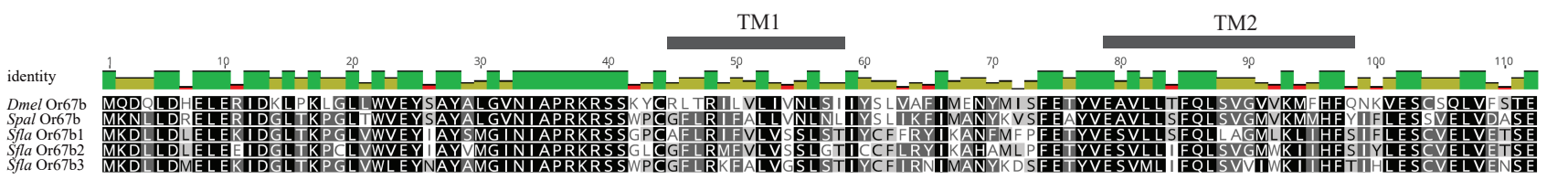

TM3

identity

Dmel Or67b

Spal Or67b
Sfla Or67b1

Sfla Or67b2
Sfla Or67b3
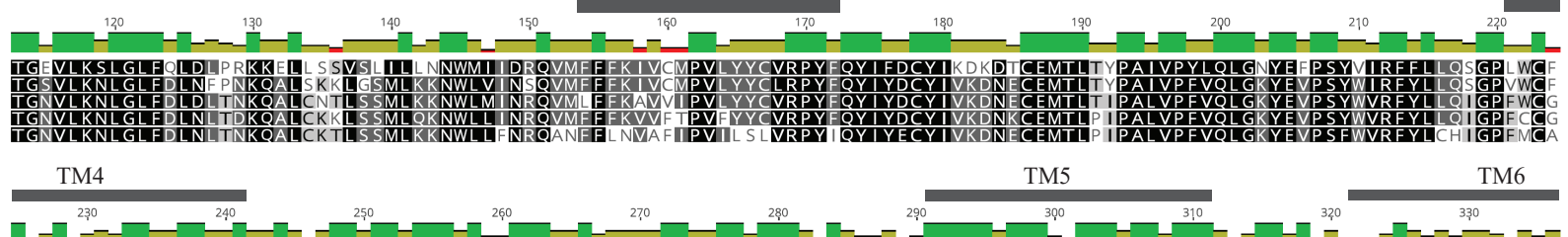

identity

Dmel 0 rgh

Shlo orb

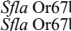

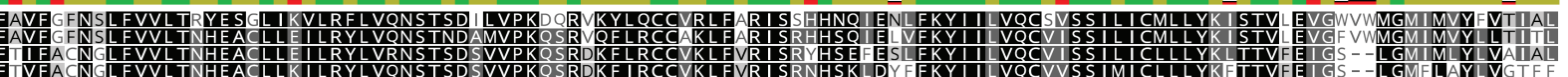

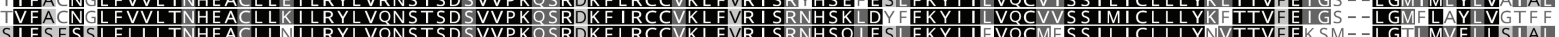

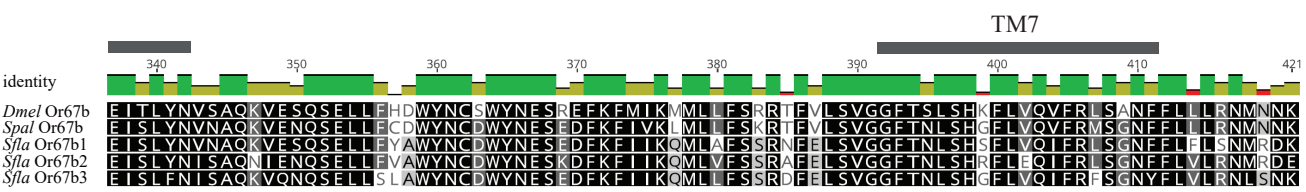


bioRxiv preprint doi: https://doi.org/10.1101/2019.12.27.889774; this version posted December 30, 2019. The cpplfighthglderfor is pleprint (which was not certified by peer review) is the author/funder, who has granted bioRxiv a license to display the prepling nulpetuitye iz made available under aCC-BY-ND 4.0 International license.

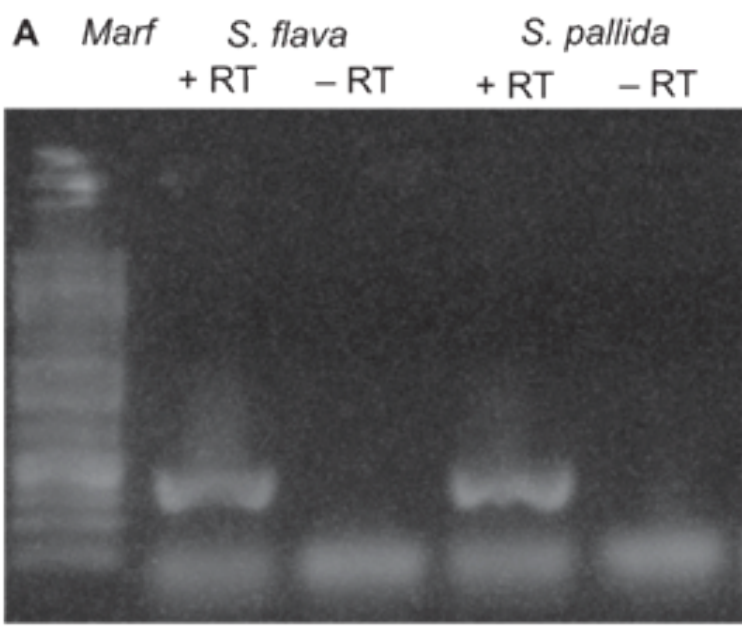
B S. pallida S. flava
Or67b Or67b1 Or67b2 Or67b3

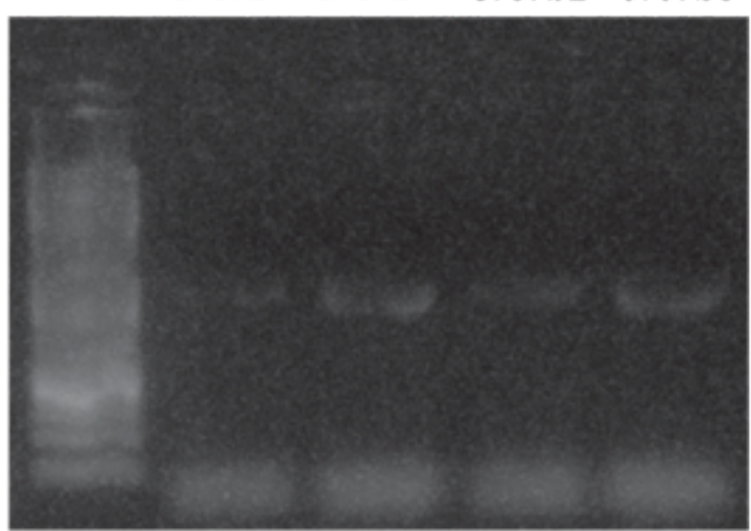


biARxiv preprint doi: https://doi.org/10.1101/2019.12.27.889774; this version posted December 30, 2019. The oppyjigtthglderfor Dis Reprint

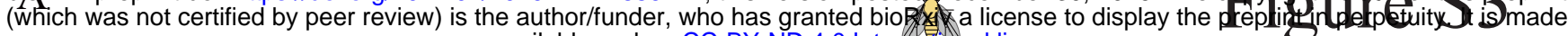
available under aCC-BY-ND 4.0 Internalional license.

empty neuron

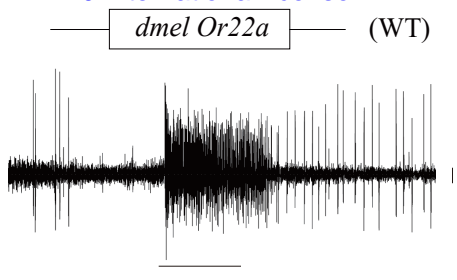

ethyl hexanoate

mineral oil

ethyl hexanoate

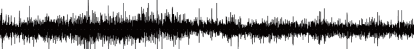

B

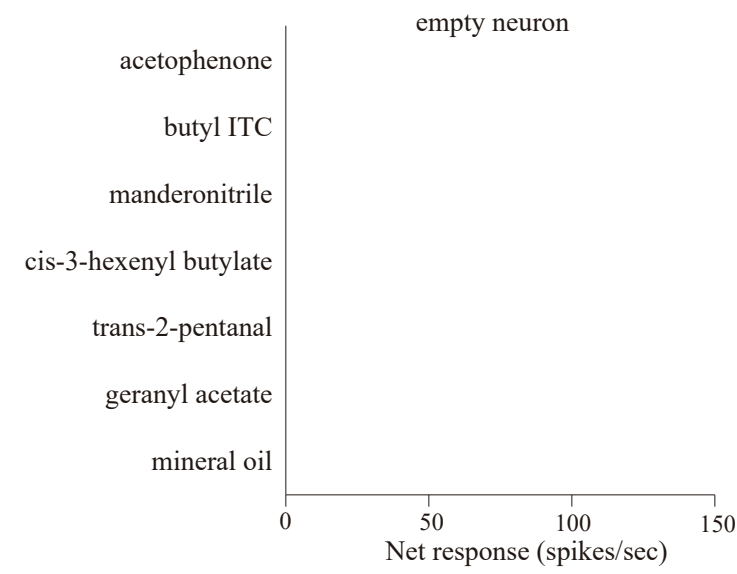

$\mathrm{C}$

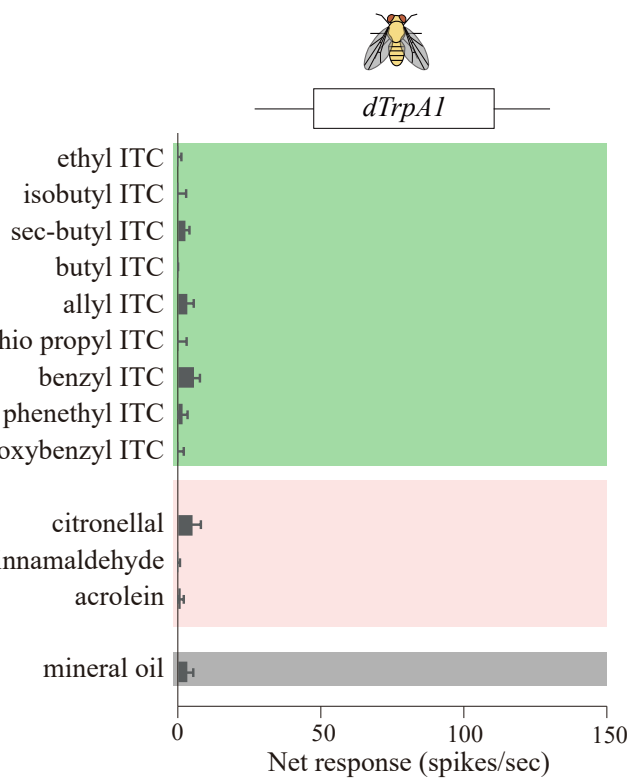




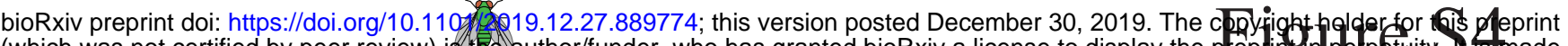
(which was not certified by peer review) is the author/funder, who has granted bioRxiv a license to display the prepling indertuity is/made

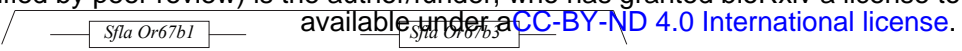

$-\mathrm{S} \sim \mathrm{NCS}$

3-methylthiopropyl ITC
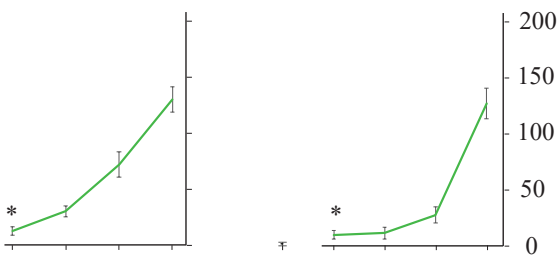

$\sim_{\mathrm{NCS}}$

butyl ITC
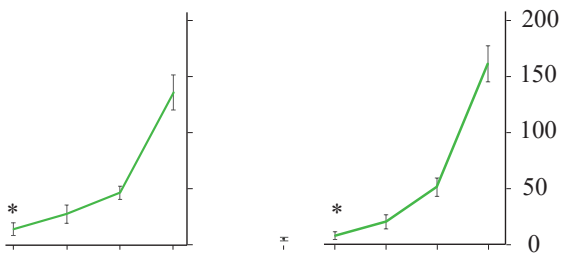

$\gamma_{\mathrm{NCS}}$

isobutyl ITC
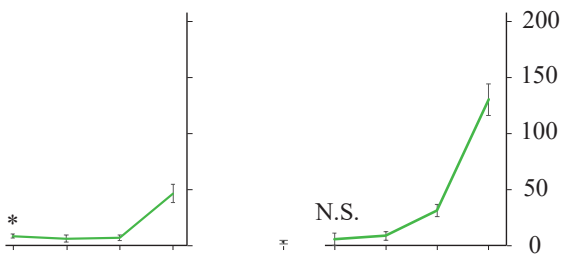

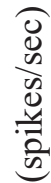

$\gamma^{\mathrm{NCS}}$

sec-butyl ITC
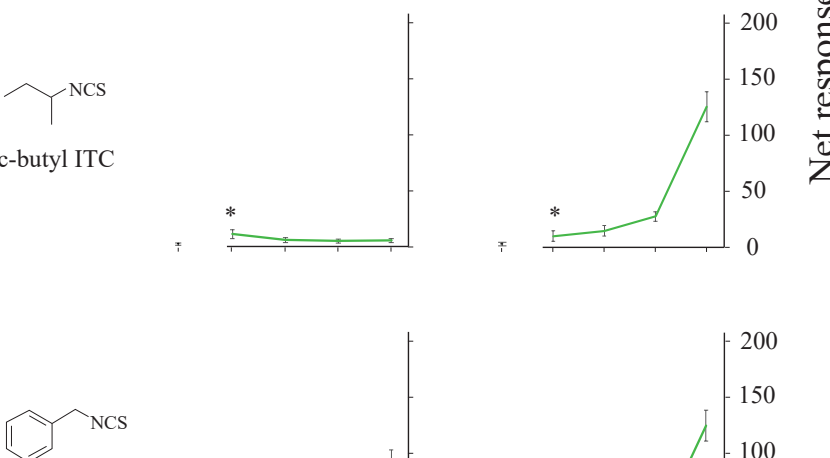

benzyl ITC
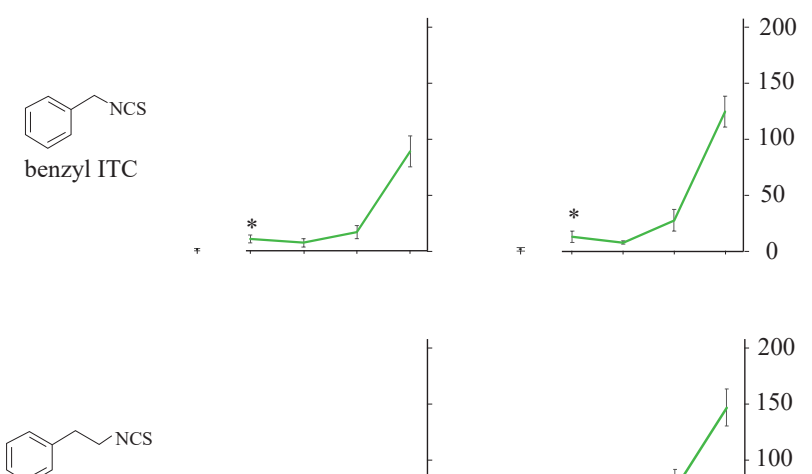

phenethyl ITC
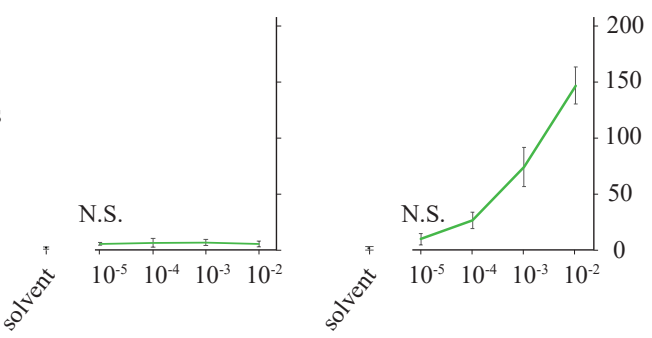


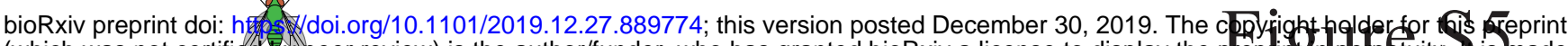

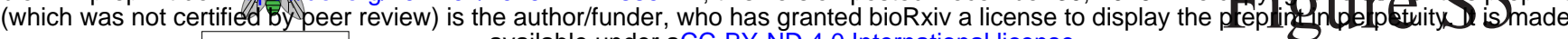
Sfla Or67b3 available under aCC-BY-ND 4.0 International license.

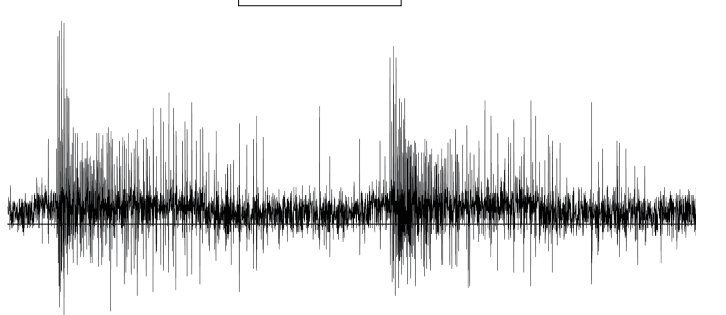

$\overline{\text { iso-butyl ITC }} \quad$ sec-butyl ITC 


\section{Supplemental Figure Legends:}

834

835

836

\section{Supplemental Figure 3:}

850 (A) Lack of Or22a expression in the ab3A neuron in the M2-MD fly line ("empty neuron" 851 mutant) was verified by lack of electrophysiological responses to ethyl hexanoate (left). In

852 contrast, in wild-type flies (Canton-S), ab3A neurons respond strongly to simulation with this

853 odor compound (right). Data indicates the net number of spikes/sec (average \pm SE, $n=6-7$ for 
854

855

856

857

858

859

860

861

862

863

864

865

866

867

868

869

870

871

872

873

874

875

each odor and control stimulation, obtained from 3 females). (B) Single odorants used for statistical analysis in Fig. 3 were tested on the mutant ab3A neuron lacking its endogenous olfactory receptor. Stimulation with any of the odorants failed to evoke spiking activity. (C) $d \operatorname{Trp} A 1$ was expressed in the ab3A neuron in the M2-MD fly line. None of the volatile ITCs or electrophiles elicited spiking activity. For a list of chemical categories see Fig. 3.

\section{Supplemental Figure 4: Odorant dose-responses of Sfla Or67b1 and Sfla Or67b3}

Responses (net spikes/sec, average +/- SE, n=6, obtained from 2 animals) of Sfla Or67b1 and Sfla Or67b3 to stimulation with increasing concentrations (vol/vol) of six different ITCs. The responses of Sfla Or67b3 steadily increased with increasing the concentration of all ITCs tested. Sfla Or67b1 and Sfla Or67b3 showed similar dose-response relationships in response to stimulation with 3-methyl-thio-propyl-ITC, butyl ITC, and benzyl ITC. Stimulation with secbutyl ITC and phenethyl ITC produced only a very slight increase in spike activity $(<10$ spikes/second) from Sfla Or67b1 at any of the concentrations tested. The asterisks indicate significant differences between the responses to the $1: 10,000 \mathrm{vol} / \mathrm{vol}$ concentration and the responses to the mineral oil control (Mann-Whitney $U$ tests corrected for multiple testing using FDR; adjusted p-values: $* \mathrm{p}<0.05)$.

\section{Supplemental Figure 5: Sfla Or67b3 has fast recovery.}

Representative electrophysiological recording obtained from the ab3 sensilla of flies expressing Sfla Or67b3 in response to stimulation with iso-butyl ITC following sec-butyl ITC with one second interval between chemical administration. The horizontal bar denotes one second and the 
876

877

878

879

880

881

882

883

884

885

886

887

888

889

890

891

892

893

894

895

vertical bar denotes $10 \mathrm{mV}$. Sfla Or67b3 sensitivity is recovered within seconds after stimulation with ITCs.

\section{Supplemental Material and Methods:}

\section{Fly husbandry and lines}

D. melanogaster (Canton-S) were reared using standard cornmeal media, yeast, and agar medium. Isofemale lines of S. pallida (collected in Berkeley, California, US) were maintained on Nutri-Fly medium (Genesee Scientific). S. flava (collected in New Hampshire, US) were maintained on fresh Arabidopsis thaliana and 10\% honey water solution. All flies were cultured at $23^{\circ} \mathrm{C}$ and $60 \%$ relative humidity under a 12-h light/12-h dark cycle. S. flava and S. pallida were about 7-10 days old at the time of experiments; D. melanogaster (wild-type or transgenic) were about 3-10 days old at the time of the experiments.

M2-MD refers to a CRISPR/Cas9 deletion of Or22a/b and a knock-in of Gal4 and DsRed by homologous repair. Gal4 is not functional but DsRed is expressed in the eye. The functional absence of $\operatorname{Or} 22 a$ and $O r 22 b$ genes in M2-MD flies was confirmed by electrophysiological analysis on $\mathrm{Or} 22 a / b$ expressing neurons in wild-type flies (Fig. S3). The M2-MD line was used to generate flies expressing Dmel Or67b, Spal Or67b and Sfla Or67b1-3 under the control of Gal4 in the ab3A "empty neuron". The UAS-SflaOr67b1, UAS-Sfla Or67b2, UAS-Sfla Or67b3, and $U A S$-Spal Or67b strains were generated during this study. 


\section{Scaptomyza Or67b gene cloning, UAS line generation, and verification of $S$. flava Or67b}

\section{transcription}

The UAS-Or67b transgene lines were constructed as follows: RNA was extracted from 20-25 male and female, laboratory-reared, adult flies of S. pallida (collected from the White Mountains, New Mexico) and S. flava (collected from near Portsmouth, New Hampshire). RNA was extracted using Trizol (Thermo-Fisher, Waltham, MA) and precipitated with isopropanol. Extracted RNA was treated with DNaseI; cDNA was then generated using qScript cDNA Supermix (Quantabio, Beverly, MA). Absence of gDNA in cDNA preparations was verified by attempting to amplify fragments of the Marf gene from reactions lacking reverse transcriptase (Fig. S2; PCR conditions and primers detailed in (59)). CDS plus 7-9 bp of untranslated sequence were amplified using High Fidelity Phusion Taq (New England BioLabs, NEB), 3\% DMSO v/v, and the PCR primers (Table S2) with the following program: initial denaturing at $98^{\circ} \mathrm{C}$ during $30 \mathrm{sec} ; 35 \mathrm{cycles}$ at $98^{\circ} \mathrm{C}$ for $10 \mathrm{sec}, 58^{\circ} \mathrm{C}$ for $30 \mathrm{sec}, 72^{\circ} \mathrm{C}$ for $45 \mathrm{sec}$, and extension at $72^{\circ} \mathrm{C}$ during $7 \mathrm{~min}$. PCR fragments of the expected size were purified using Qiaquick Gel purification kit protocol (Qiagen). An overhang was added to purified Or67b amplicons with Taq polymerase (Fermentas) and cloned using the pGEM-T Easy cloning kit protocol (Promega). Plasmids were extracted and purified using the GenElute plasmid miniprep kit (Sigma-Aldrich, St. Louis, MO). EcoRI and KpnI cut sites were introduced using restriction enzyme cut-site primers (Table S2) with $10 \mathrm{ng} / \mu \mathrm{L}$ diluted plasmids (as template) with 3\%

DMSO vol/vol and the following program: initial denaturing at $98^{\circ} \mathrm{C}$ for $30 \mathrm{sec} ; 35$ repetitions of $98^{\circ} \mathrm{C}$ for $10 \mathrm{sec}, 55^{\circ} \mathrm{C}$ for $50 \mathrm{sec} ; 72^{\circ} \mathrm{C}$ for $45 \mathrm{sec}$; and final extension at $72^{\circ} \mathrm{C}$ for $7 \mathrm{~min}$. The pUAST attB plasmid (60) and the four S. pallida and S. flava Or67b PCR amplicons with RE flanking sites were individually double-digested with KpnI and EcoRI high-fidelity enzyme in 
cut smart buffer for 3 hours, according to the manufacturer's protocol (NEB). Cut fragments were gel-purified using the Qiaquick Gel Cleanup Kit (Qiagen) and ligated in a 1:3 vector:insert molar ratio using T4 ligase (Promega). Ligations were transformed into JM109 cells. Some cells were preserved as glycerol stocks and a portion were sent for injection into the $\mathrm{y}^{1} \mathrm{w}^{67 \mathrm{c} 23}$; $\mathrm{P}\{$ CaryP $\}$ attP2 fly line (BestGene Inc., Houston, Texas, USA). Transformants were selected from individually injected flies with eye color rescue phenotypes. The four $U A S-O r 67 b$ lines created in this study, the UAS-Dmel Or67b line, and the $U A S$-dTrpAl (stock no. 26264) line was each crossed into the M2-MD line. The progeny were then used for SSR recordings and behavioral experiments.

\section{Behavioral tests of olfactory attraction}

The olfactory responses of mated, non-starved D. melanogaster (Canton-S or transgenic), S. pallida, and S. flava were tested using a dual-choice "Y-shaped" olfactometer based on one previously published (20). The "Y piece" of the olfactometer was a propylene connector (43 $\mathrm{mm}$ long x $60 \mathrm{~mm}$ ); the open ends of the arms of the "Y" were each connected to a 1-ml syringe containing a piece of filter paper $(600 \times 50 \mathrm{~mm})$ loaded with the odor or control stimuli. Charcoal-filtered air was delivered to each of the two syringes using silicon tubing at 250 $\mathrm{ml} / \mathrm{min}$; thus, at the base of the maze air flow was approximately $500 \mathrm{ml} / \mathrm{min}$. Two hours (in the case of $D$. melanogaster and $S$. pallida) or 20 hours before tests (in the case of $S$. flava) insects were gently anesthetized under $\mathrm{CO}_{2}$ and placed in groups of four in open-top and mesh-bottom cylindrical release containers (20 mm long x $10 \mathrm{~mm}$ diameter) constructed using silicon tube.

The open top of the containers was capped with a piece of cotton soaked in distilled water (in the case of $D$. melanogaster and $S$. pallida) or with a piece of cotton soaked in $10 \% \mathrm{vol} / \mathrm{vol}$ aqueous 
942 honey solution (in the case of $S$. flava). Before tests, each release tube was placed in ice during

943 45-60 seconds to slow down insect activity, the cotton cap was removed, and the open-top of the

944 tube was carefully slid into the open end of the Y maze. Thus, upon being released, insects could

945 walk upwind towards the "decision point" (intersection of the short and long arms of the "Y")

946 and turn towards either the odor-laden or the odorless arm of the maze. Although four insects

947 were released at once (to increase experimental efficacy), only the first choice (and the time of

948 the choice) was recorded; a choice was considered as such only if the insect walked past at least

$94910 \mathrm{~mm}$ into one of the arms, orienting upwind. The test was discarded if two or more insects

950 choose the two arms of the maze within a 3-second window of each other. Each test lasted a

951 maximum of five minutes, and each group of insects was used only once. As much as possible,

952 insects from the same cohort were tested in the same day with different odors/odorants; insects

953 from the three different species were also, as much as possible, tested in the same day with a

954 given odor/odorant/different fly genotypes. Tests with each species/stimulus were conducted in

955 at least five different days to compensate for possible day-to-day variations. Results from an

956 individual test session with a given odor/odorant were discarded if insects did not make a choice

957 is more than $50 \%$ of tests (this happened in less than $5 \%$ of experimental sessions). The position

958 of the odor and odorless arms was switched every 1-2 tests to control for positional asymmetries;

959 the mazes and odor sources were changed and replaced for clean/new ones every four tests or 10

960 minutes, whichever comes first.

961 The odor/odorant was loaded in a piece of filter paper and inserted into the 1-ml syringe

962 right before tests; control syringes had a piece of filter paper (3 x $40 \mathrm{~mm})$ loaded with the

963 mineral oil solvent (for odorant solutions) or water (for odor mixtures). Experimental and control

964 filter papers were replaced by fresh ones every 4 tests or 10 minutes, whichever came first. The 
965 odorants (20 $\mu \mathrm{l}$ of 1:100 vol/vol mineral oil solution) used in experiments were butyl ITC

966 (Sigma-Aldrich, CAS \# 592-82-5, USA) and sec-butyl ITC (Sigma-Aldrich, CAS \# 15585-98-5,

967 USA). For tests with transgenic flies, responses were tested at 1:100 vol/vol and 1:1,000 vol/vol

968 of butyl ITC. We also used apple cider vinegar (40 $\mu$ l, O Organics, USA; $40 \mu$ l of distilled water

969 was as control stimulus in these tests). For tests of host-orientation, two-four leaves from young

970 arugula plants grown in an insect and insecticide/pesticide free chamber or greenhouse were

971 excised just before tests, and placed in 5-ml syringes connected to the Y-maze; control syringes

972 had two pieces of tissue paper. In all cases the Y-mazes, tubing and syringes were cleaned with

$97370 \%$ ethanol and allowed to air-dry before reusing. Experiments were conducted during the $2^{\text {nd }}$ -

$9745^{\text {th }}$ hour of the insects' photophase at $24^{\circ} \mathrm{C}$ under white light (Feit electric, 100 Watts; in the case

975 of S. pallida and D. melanogaster) or green light (Sunlite green, 100 Watts; in the case of

976 experiments with $S$. flava). A total of 1,061 (669 with wild-type flies of D. melanogaster, $S$.

977 pallida and S. flava; 392 with transgenic D. melanogaster) tests were and insects made a choice 978 in $84 \%(888)$ of tests.

979 For each odor/odorant, species, sex and genotype, the number of tests with the first insect 980 orienting towards the odorous arm and the odorless arm of the Y-maze were tested against a 50\% 981 expected random distribution using two-tailed Binomial tests (48). P-values were adjusted for multiple comparisons using the false discovery rate (FDR) method of Benjamini-Hochberg (49);

983 results were considered significant if the Benjamini-Hochberg adjusted p-value was $<0.05$. For 984 all tests, we verified that the power was $>0.8$. 


\section{Molecular phylogeny of drosophilid Odorant Receptors $($ Or $)$ :}

Translations of Ors from D. grimshawi, D. mojavensis, D. virilis and D. melanogaster (builds dgri r1.3, dmoj r1.3, dvir r1.07 and dmel r6.28, respectively) were downloaded from Flybase (www.flybase.org, (50)). S. flava sequences were previously published (19). Three hundred and nine sequences were aligned in MAFFT v7.017 with the E-INS-I algorithm and then manually adjusted (51). Models were fitted to the alignment using IQ-Tree and tested using the AIC criterion (52). A maximum likelihood (ML) phylogeny was generated using the Or protein alignment in RAxML v8.2.10 with the CAT model of rate heterogeneity with seven distinct categories, the JTT substitution matrix, empirical amino acid frequencies, and 1,000 rapid bootstraps (53). Orco sequences were designated as the outgroup.

\section{Molecular phylogeny of drosophilid Or67b genes:}

Or67b CDS from D. grimshawi, D. mojavensis, D. virilis D. sechellia, D. simulans, D. erecta, D. yakuba, D. pseudoobscura, D. persimilis, D. ananassae, D. willistoni and D. melanogaster (builds dgri r1.3, dmoj r1.3, dvir r1.07, dsec r1.3, dsim r1.4, dere r1.3, dyak r1.3, dpse r3.2, dper r1.3, dana r1.3, dwil r1.3 and dmel r6.28, respectively) were downloaded from Flybase (www.flybase.org, (50)). The $S$. pallida DNA sequence was obtained through PCR as described above; S. flava DNA sequences were previously published (19). Sequences were aligned, models fitted and chosen according to AIC $(\mathrm{GTR}+\mathrm{I}+\mathrm{G})$ in IQ-Tree (52). Trees were inferred using RAxML (v8.2.10) with the GTRCATI model and 1000 rapid bootstraps, and MrBayes (v3.2.6) setting Nst to 6, nucmodel to 4by4, rates to Invgamma, number of generations to 125,000 , burnin equal to $20 \%$ of generations, heating to 0.2 , number of chains to 4 , runs to 2 and priors set to default setting (54). 


\section{Analysis of molecular evolution:}

CDS of homologs of every Or gene in S. flava found in the 12 Drosophila genome builds were

1011 aligned to $S$. flava Or CDS. Homology was assessed according to inclusion in well supported clades in the $O r$ translation phylogeny above; S. flava sequences were previously published (19).

1013 Sequences were aligned in MAFFT (v7.017) (51) and adjusted manually to preserve codon

1014 alignments. Or98a-like genes found in subgenus Drosophila species were split into three

1015

1016

1017

1018

1019

1020

1021

1022

1023

1024

1025

1026

1027

1028

1029

1030 separate clades, as were a group of $0 r 83 c$ paralogs not found in D. melanogaster, and a group of Or85a-like genes. All sequences examined of Or46a contain two alternatively spliced exons, so this gene was analyzed with all gene exon sequences in a single alignment as single taxa. Or69a, however, contains alternatively spliced exons only among Sophophora species. These alternative splice forms were analyzed as separate taxa. Phylogenies were generated for every alignment using PhyML (55) with the GTR + G substitution models. If these trees showed $>70 \%$ bootstrap support for a topology contrary to the known species topology, or if the Or homology group contained duplicates, these trees were used in PAML analyses instead of the species tree. Branch models of sequence evolution were fit using PAML 4.9h (26). A foreground/background branch model was fit for every $S$. flava tip branch and every ancestral branch in a Scaptomyza-specific

Or gene duplication clade, and compared in a likelihood ratio test to a null model with one $\mathrm{d} N / \mathrm{d} S$ rate for every unique phylogeny (75 tests total). After focusing on $0 r 67 b$, patterns of molecular evolution among the drosophilid Or67b homologs were explored using the expanded Or67b CDS phylogeny above. Foreground/background branch models were fit for every branch in the Or67b phylogeny with identical likely ratio tests performed as above (29 tests total Fig. 2B; Table S1). P-values were adjusted for multiple-testing using the FDR method (49). 


\section{Distance tree obtained from responses of ab3A neurons expressing Or67b transgenes}

1032

1033

1034

1035

1036

1037

1038

1039

1040

1041

1042

1043

1044

1045

1046

1047

1048

1049

1050

1051

1052

1053

A matrix of average response of five $O r 67 b$ transgene receptors to 44 odorant compounds (1:100

vol/vol concentration) was produced (Table S3). Net responses were calculated by subtracting

the response to mineral oil or dimethyl sulfoxide solvent from the odor-evoked responses. A

Euclidean distance matrix was generated using the dist function of the R stats package(56).

Receptor responses were clustered by using the neighbor joining (NJ) algorithm on this distance

matrix. Support for clusters was assessed using 1000 bootstraps of the original response matrix

by generating distance matrices and NJ trees on the pseudo-datasets in ape (v5.3) (57).

\section{Single sensillum recordings (SSR)}

Female fed flies were prepared for SSR as previously described (58). We identified sensilla of targeted olfactory receptor cells using an Olympus BX51WI upright microscope with 10x and 50x objectives (Olympus, UPlanFL N 10x, UPlanFL N 50x). Extracellular activity was recorded by inserting a tungsten electrode into the base of the ab3 sensillum. Signals were amplified 10,000 x (A-M systems, Differential AC Amplifier model 1700), digitized using a 16-bit analogdigital converter, filtered (low cut-off: $300 \mathrm{~Hz}$, high cut off: $3 \mathrm{k} \mathrm{Hz}$ ), and analyzed off-line with WinEDR (v3.9.1; University of Strathclyde, Glasgow). A tube delivering a constant flow of charcoal-filtered air was placed near the fly's head, and the tip of the stimulation pipette (50 ml) was inserted into the constant air stream. The stimulation pipette contained a piece of filter paper loaded with $20 \mu \mathrm{l}$ of odorant solution or the solvent control. One second pulse of clean air was delivered to the stimulus pipette using a Stimulus Controller CS 55 (Syntech, Germany). We used three standard odors for identification of ab3 sensilla (all from Sigma-Aldrich, US, purity $>$ 1\%): ethyl hexanoate (CAS \# 123-66-0), ethyl acetate (CAS \# 141-78-6) and 2-heptanone (CAS 
1054 \# 110-43-0). The following odor sources (all purchased in Berkeley, California, USA unless

1055 otherwise mentioned; $20 \mu \mathrm{l}$ of material loaded on filter paper) were used: apple cider vinegar (40

$1056 \mu 1$, O Organics, USA), grated roots of Wasabia japonica (wasabi), organic roots of Armoracia

1057 rusticana (horseradish), Brassica rapa (turnip), Raphanus sativus (daikon), and Beta vulgaris

1058 (beet). Eruca vesicaria (arugula) was grown from seeds at $23^{\circ} \mathrm{C}$ and $60 \%$ relative humidity in a

1059 12-hours light: 12-hours dark cycle, and leaves from 3-8 weeks old plants were used for odor

1060 stimulation. Roots and leaves were grated and homogenized using a vegetable grater to a volume

1061 equivalent to $\sim 500 \mu 1$. The following odorants (all from Sigma-Aldrich, US) were diluted 1:100

1062 vol/vol in dimethyl sulfoxide: iodoacetamide (CAS \# 144-48-9), N-methylmaleimide (CAS \#

1063 930-88-1), N-hydroxysuccinimide (CAS \# 6066-82-6), and 3-indol-carbinole (CAS \# 700-06-1)

1064 and benzyl thiocyanate (CAS \# 3012-37-1). All the other chemicals were diluted in mineral oil at

$10651: 100,1: 1,000$, and 10,000 vol/vol. The "net number of spikes" were obtained by counting the

1066 number of spikes during a 1-second window 0.2 seconds after the onset of stimulation, and

1067 subtracting from this number the background spiking activity (obtained by counting the number

1068 of spikes in a 1-second window prior to the onset of the odor stimulation). Data was analyzed

1069 using Mann-Whitney Rank sum tests (for comparisons involving two means) and p-values were

1070 adjusted for multiple comparisons using the FDR method, or by Kruskal-Wallis ANOVAs

1071 followed by Dunn's tests (for comparisons involving more than two means).

1073 Principal Component Analysis (PCA). For visualization, Principal component analysis (PCA)

1074 was done using Python. 


\section{Data Analysis and figure generation:}

1077 All images and drawings are originals prepared by the authors. Figures were prepared via a

1078 combination of WinEDR (v3.9.1), R Studio (v1.2.1335), Microsoft Excel (2016), Adobe

1079 Illustrator (2019), Python, and Geneious (10.0.9). 